ThE Astrophysical Journal, 343:369-392, 1989 August 1

(c) 1989. The American Astronomical Society. All rights reserved. Printed in U.S.A.

\title{
THEORETICAL STUDIES OF THE INFRARED EMISSION FROM CIRCUMSTELLAR DUST SHELLS: THE INFRARED CHARACTERISTICS OF CIRCUMSTELLAR SILICATES AND THE MASS-LOSS RATE OF OXYGEN-RICH LATE-TYPE GIANTS
}

\author{
W. A. SChutTE \\ Laboratory Astrophysics Group, Rijks Universiteit, Leiden; and Space Science Division, NASA/Ames Research Center \\ AND \\ A. G. G. M. TIELENS \\ Space Science Division, NASA/Ames Research Center; and Space Sciences Laboratory, University of California, Berkeley \\ Received 1987 November 24; accepted 1989 January 4
}

\begin{abstract}
We have modeled the infrared emission of spherically symmetric, circumstellar dust shells with the aim of deriving the infrared absorption properties of circumstellar silicate grains and the mass-loss rates of the central stars. As a basis for our numerical studies, a simple semianalytical formula has been derived that illustrates the essential characteristics of the infrared emission of such dust shells. A numerical radiative transfer program has been developed and applied to dust shells around oxygen-rich late-type giants. Free parameters in such models include the absorption properties and density distribution of the dust. An approximate, analytical expression is derived for the density distribution of circumstellar dust driven outward by radiation pressure from a central source. A large grid of models has been calculated to study the influence of the free parameters on the emergent spectrum. These results form the basis for a comparison with near-infrared observations.

Observational studies have revealed a correlation between the near-infrared color temperature, $T_{c}$, and the strength of the $10 \mu \mathrm{m}$ emission or absorption feature, $A_{10}$. This relationship, which essentially measures the near-infrared optical depth in terms of the $10 \mu \mathrm{m}$ optical depth, is discussed. Theoretical $A_{10}-T_{c}$ relations have been calculated and compared to the observations. The results show that this relation is a sensitive way to determine the ratio of the near-infrared to $10 \mu \mathrm{m}$ absorption efficiency of circumstellar silicates. These results as well as previous studies show that the near-infrared absorption efficiency of circumstellar silicate grains is much higher than expected from terrestrial minerals. We suggest that this enhanced absorption is due to the presence of ferrous iron $\left(\mathrm{Fe}^{2+}\right)$ color centers dissolved in the circumstellar silicates. By using the derived value for the ratio of the near-infrared to $10 \mu \mathrm{m}$ absorption efficiency, the observed $A_{10}-T_{c}$ relation can be calibrated in terms of the total dust column density of the circumstellar shell and thus the mass-loss rate of late-type giants can easily be derived.

Detailed models have been made of the infrared emission of three well-studied Miras: R Cas, IRC 10011, and $\mathrm{OH} 26.5+0.6$, with the emphasis on the shape of the $10 \mu \mathrm{m}$ emission or absorption feature. The results show that the intrinsic shape of the $10 \mu \mathrm{m}$ resonance varies from a very broad feature in $\mathrm{R}$ Cas to a relatively narrower feature in $\mathrm{OH} 26.5+0.6$, with IRC 10011 somewhere in between. Possible origins of this variation are discussed. The mass-loss rates from these objects are calculated to be $3 \times 10^{-7}, 2 \times 10^{-5}$, and $2 \times 10^{-4} M_{\odot} \mathrm{yr}^{-1}$ for $\mathrm{R}$ Cas, IRC 10011 , and $\mathrm{OH} 26.5+0.6$, respectively. These results are compared to other determinations in the literature.

Subject headings: infrared: spectra - stars: circumstellar shells - stars: late-type
\end{abstract}

\section{INTRODUCTION}

The stellar pulsations of stars on the asymptotic giant branch (AGB stars) drive strong shock waves into the photosphere which transport mass to large distances $\left(\sim 5 r_{*}\right)$ where condensation of dust particles takes place (Hinkle, Hall, and Ridgway 1982; Wood 1979; Wilson and Hill 1979). Radiation pressure by stellar photons accelerates these dust grains outward. Gas-grain collisions transfer this momentum to the gas and it is dragged along (Kwok 1975; Tielens 1983). In this way an outwardly expanding, circumstellar dust and gas shell is formed. The radiation of the central source is absorbed by the dusty envelope and reemitted in the form of thermal dust radiation. The maximum temperature of the dust in the circumstellar shell is about $1000 \mathrm{~K}$-the condensation temperature of the dust at the inner radius-and decreases outwardly due to geometrical dilution of the radiation field. Therefore, these objects show an infrared excess above the photospheric emission beyond about $3 \mu \mathrm{m}$. Indeed, observations have shown that infrared emission is a key observational characteristic of such objects. The infrared properties of such shells show a wide variety, ranging from objects with only slight infrared excesses (i.e., the optically thin shells around Miras) to those that are totally obscured by their shells and emit virtually all their radiation at infrared wavelengths (i.e., the optically thick shells around OH/IR stars).

The shape of the emergent infrared spectrum depends to a large extent on the absorption properties of the circumstellar dust. Conversely, the observed spectra can thus be used to derive these dust properties. For example, the grains in the circumstellar envelope of oxygen-rich giants consist of an amorphous silicate material as judged from the presence of broad, structureless 10 and $20 \mu \mathrm{m}$ emission or absorption features due to the $\mathrm{SiO}$ stretching and bending vibrations in their spectra (Merrill 1977). Moreover, previous theoretical model- 
ing of such envelopes has shown that the near-infrared ( $\lesssim 6 \mu \mathrm{m}$ ) absorption cross section of these circumstellar silicates has to be higher than expected from terrestrial minerals (Jones and Merrill 1976; Bedijn 1977). This analysis was based on a relatively small data set consisting mainly of fairly optically thin $(\tau[10 \mu \mathrm{m}] \lesssim 3)$ Miras and IRC sources, and only a rather qualitative conclusion could be derived. In this paper this question is reinvestigated based on an observed correlation between near-infrared color temperature, $T_{c}$, and $10 \mu \mathrm{m}$ extinction optical depth, $A_{10}$, in a large data set, $(\sim 35$ objects $)$ including many OH/IR stars. Their high near-infrared optical depth renders them very useful for such an analysis since the emergent spectrum is much less sensitive to other free parameters, such as the stellar effective temperature and the inner boundary conditions.

Previous studies have also shown that the intrinsic shape of the $10 \mu \mathrm{m}$ silicate feature can vary from source to source (Mitchell and Robinson 1981; Rogers, Martin, and Crabtree 1983; Pégourie and Papoular 1985; Schutte and Tielens 1985). These variations probably reflect a temperature dependence of the intrinsic absorption properties of the $\mathrm{Si}-\mathrm{O}$ stretching mode in silicates due to, for example, thermal shrinking of the lattice (Tielens and Allamandola 1987a). Alternatively, these variations could be caused by variations in the condensation history of the grains (Schutte and Tielens 1985). In particular, variations in the condensation temperature may result in differences in the degree of disorder and thus in the shape of the $10 \mu \mathrm{m}$ absorption feature (Tielens and Allamandola 1987a). Detailed analysis of $10 \mu \mathrm{m}$ spectroscopy of many sources with different physical conditions are required to unravel these possibilities. In this paper, we study the detailed shape of the $10 \mu \mathrm{m}$ feature in three late-type giants whose mass-loss rates differ by several orders of magnitude. Recently, the shape of the 10 and $20 \mu \mathrm{m}$ silicate emission features in circumstellar dust shells with low $10 \mu \mathrm{m}$ optical depth has been studied using the LRS spectra in the IRAS data base (Gal et al. 1987). Observations obtained with the IRAS data base have also been modeled by Bedijn (1987) and Volk and Kwok (1988).

Once the infrared properties of the emitting silicate grains have been determined, the total dust column density as well as the inner radius of the dust shell can be estimated from model fits to observed infrared spectra. Using observed outflow velocities from $\mathrm{CO}$ or $\mathrm{OH}$ observations, this permits the determination of the mass-loss rate of the central star. Since mass loss from late-type giants is an important source for replenishing the gas and dust in the interstellar medium (Jura 1987), models of the infrared emission of such objects can be used to constrain theories of the evolution of the interstellar medium. In particular, the amount of grain material lost by these objects is an essential boundary condition for theories concerning the destruction and formation of interstellar dust (Dwek and Scalo 1980; Seab 1987; McKee et al. 1987). For many moderately obscured $(\tau[10 \mu \mathrm{m}]<10) \mathbf{M}$ giants and supergiants in the AFGL catalog, detailed analysis of the observed IR spectra have been performed by Rowan-Robinson and Harris (1982, 1983), and the derived optical depth of the dust in the circumstellar shell can be converted into mass-loss rates. Here we analyze the observed $A_{10}-T_{c}$ relation and derive mass $\cdot$ loss rates for about 35 stars, including many heavily obscured OH/IR stars $(\tau[10 \mu \mathrm{m}]>10)$. The $A_{10}-T_{c}$ relation proves to be a relatively simple way to derive reliable mass-loss rates from IR observations.

To model the infrared emission of circumstellar dust shells the equation of radiative transfer must be solved. The modeling of dust-enshrouded objects presented in this paper is based on the "diffusion" method described by Leung $(1975,1976 a, b)$. This method yields an exact solution since no terms in radiative transfer equation are neglected in the numerical procedure. Using this method, an extensive study of the free parameter space has been made. In particular, we have studied the influence of variations in the absorption properties of the dust as well as the inner boundary conditions. We have also included a more realistic dust density distribution than previous studies, which takes the acceleration near the inner edge approximately into account. In this paper we restrict ourselves to an analysis of the near- and mid-infrared (1-15 $\mu \mathrm{m})$ emission from circumstellar dust shells with the aim of deriving the optical properties of circumstellar silicates as well as the stellar mass-loss rate.

In $\S$ II we describe the main features of circumstellar dust shells around Mira variables. This includes a discussion of the structure of such dust shells as revealed by recent observations. The determination of the absorption properties of circumstellar silicates from observed IR spectra is essentially an inversion problem. In the optically thin limit this inversion is relatively simple. This is also discussed in $\S$ II. In $\S$ III the numerical radiative transfer method is discussed, and the assumed optical properties of the dust are described. A simple analytical expression for the dust density distribution is also derived, which takes the radiative acceleration approximately into account. A large grid of models has been calculated and the emergent spectra are presented and discussed in Appendix A. Observations show that the near-infrared color temperature, $T_{c}$, and the strength of the $10 \mu \mathrm{m}$ (emission or absorption) feature $A_{10}$, are correlated. This observed relationship is discussed in $\S$ IV. It reflects the relation between the near-infrared and $10 \mu \mathrm{m}$ optical depth, and comparison with numerical model studies allows the near-infrared absorption efficiency relative to that at $10 \mu \mathrm{m}$ to be determined. The optical and near-infrared absorption of silicate grain materials is briefly examined, and it is suggested that electronic transitions of dissolved transition metals may be responsible for the high near-infrared absorption of circumstellar silicates. Finally, the $A_{10}-T_{c}$ relation is calibrated in terms of the circumstellar dust column density and mass-loss rates are derived for our sample of OH/IR stars. In $\S \mathrm{V}$, detailed model fits to the infrared observations of three late-type giants losing mass (e.g., R Cas, IRC 10011, and $\mathrm{OH}$ $26.5+0.6)$ are presented. The "best fit" optical properties of the circumstellar silicate grains are determined and shown to vary from object to object. These variations are also discussed. Finally, mass-loss rates are determined for these objects and compared to values in the literature. The results of our studies are summarized in $\S \mathrm{VI}$.

\section{MAIN FEATURES OF DUST IN CIRCUMSTELLAR ENVELOPES}

\section{a) The Structure of the Envelope}

The circumstellar envelope of late-type giants losing mass consists of three distinct regions (Hinkle, Hall, and Ridgway 1982; Hinkle, Scharlach, and Hall 1984; Hinkle 1985). First, there is the regularly pulsating stellar photosphere. The general oscillation of the stellar interior drives a strong, outwardly propagating shock into the atmosphere around maximum light. This shock transports material to large distances from the stellar surface $\left(\sim 5 r_{*}\right)$ where it becomes part of the second distinct region, the (quasi)-stationary layer. The gas tem- 
perature is fairly low in this region $(\sim 800 \mathrm{~K})$, and dust particles condense in this layer. Radiation pressure by stellar photons accelerates these grains outward. Because of gas-grain collisions the gas is dragged along. In this way the third region-an extended, outwardly expanding, circumstellar shell-is formed.

The complexity of the circumstellar envelope is reflected in the spectrum of absorption lines and emission lines. For example, each of the three regions can be studied in $\mathrm{CO}$ absorption lines (Hinkle, Hall, and Ridgway 1982). The hydrogen emission lines originate, however, in the shocked gas (Willson 1976), while the metallic absorption lines are formed in material that is trickling back from the stationary layer to the photosphere (Hinkle, Hall, and Ridgway 1982). The peak velocity as well as the absence of violent variations places the $\mathrm{SiO}$ maser in the cool stationary layer (Hinkle, Scharlach, and Hall 1984), despite the large excitation energies required for this maser. The infrared dust continuum, the molecular rotational lines, as well as the $\mathrm{H}_{2} \mathrm{O}$ and $\mathrm{OH}$ maser, are formed in the cool expanding shell. This interpretation is supported by detailed theoretical models of shock wave propagation in pulsating red giant atmospheres (Willson and Hill 1979; Willson 1987; Wood 1979; Bowen and Beach 1987; Bowen 1988).

For our theoretical study of radiation transfer in the circumstellar envelope of Miras and OH/IR stars, the only region of importance is the cool expanding shell. Once the initial dust condensation nuclei have formed, radiation pressure rapidly accelerates them to their local terminal velocity (Kwok 1975; Tielens 1983). Therefore, on the inner edge, the expanding dust shell is separated from the stationary layer by a very thin acceleration zone, in which the relative dust abundance and grain size and thus the opacity drops very rapidly to zero. Over most of the envelope the dust moves at its terminal velocity. Assuming a constant mass-loss rate, the dust density distribution is then inversely proportional to the distance squared, except in the condensation and acceleration zone where the density distribution is more complex. Assuming that the dust condensation is instantaneous, the density distribution in the acceleration zone will be further investigated in $\S$ III $c$.

The conditions that set the inner boundary of the dust shell are expected to be of some importance for the interpretation of observed IR spectra as well as for their variations with phase. The condensation conditions of dust in circumstellar shells are however quite uncertain. Thermodynamic equilibrium calculations of a cooling gas with cosmic composition show that calcium-aluminum silicates are the first to condense out at about $1400 \mathrm{~K}$. The majority of the silicon is expected to condense in the form of magnesium silicates at about $1050 \mathrm{~K}$ (Hackwell 1971). However, the concept of thermodynamic equilibrium condensation is probably not a very viable one for dust formation in circumstellar shells (Nuth and Donn 1985; Tielens 1983). In particular, the large width and absence of substructure in the $10 \mu \mathrm{m}$ feature suggest an amorphous structure for the condensing silicates (Day 1976; Day and Donn 1978). Probably, the silicate grains have therefore condensed out under highly supercooled conditions (Tielens and Allamandola 1987a). That is, the dust temperature in the condensation zone is much less than expected from thermodynamic considerations. In fact, it is quite likely that dust condensation is limited by the survivability of the condensation nuclei in the extended photosphere and stationary layer (Tielens 1983). Rather than taking the dust condensation temperature as the inner boundary condition, as is commonly done in radiative transfer studies of this kind, the influence of the inner boundary condition on the emergent spectrum will be examined in more detail (see also Martin and Rogers 1987).

\section{b) The Optically Thin Limit}

The aim of this paper is to derive the optical properties of the newly formed silicates in the envelopes of Miras and the closely related $\mathrm{OH} / \mathrm{IR}$ stars from their observed spectra. This requires knowledge of the dust temperature gradient in the circumstellar shell. It may be instructive to consider first the optically thin case, in which the dust is heated only by the direct starlight (e.g., the optically thin approximation). Approximating the stellar flux by a blackbody at temperature $T_{\text {eff }}$, the dust energy balance yields the temperature $T_{d}$, at a distance $r$ from the star,

$$
T_{d}=c\left[\left(Q_{\mathrm{abs}} / Q_{\mathrm{em}}\right) T_{\mathrm{eff}}^{4}\left(r_{*} / r\right)^{2}\right]^{1 / 4},
$$

where $c$ is a constant, $r_{*}$ is the stellar radius, and $Q_{\mathrm{abs}}$ and $Q_{\mathrm{em}}$ are the dust absorption and emission efficiencies averaged over the Planck function at the effective temperature and dust temperature, respectively. In general, these efficiencies are temperature-dependent as well. Assuming a $\lambda^{0}$ (gray body), a $\lambda^{-1}$, and a $\lambda^{-2}$ dependence for the absorption and emission yields the familiar result $T_{d} \sim r^{-2 / n}$, with $n$ equal to 4,5 , and 6 , respectively. Although silicates show strong vibrational modes at 10 and $20 \mu \mathrm{m}$, for temperatures below $200 \mathrm{~K}$ these are not excited, and $n$ is between 5 and 6 depending on the structure of the silicate (layer-lattice or three-dimensional silicates; Tielens and Allamandola 1987a; Tielens 1987). Between 200 and $1000 \mathrm{~K}$ the emission is dominated by the 10 and $20 \mu \mathrm{m}$ vibrational modes, and the Planck-averaged absorption efficiency is fairly independent of the dust temperature $(n=4$; i.e., a gray body; Draine and Lee 1984).

Generally, it is assumed that at the inner radius, $r_{0}$, the dust is formed at a temperature equal to the sublimation temperature, $T_{s}$, of the material ( $\sim 1000 \mathrm{~K}$ for silicates). In the optically thin case the temperature structure of the envelope is then fixed, that is

$$
T_{d}(r)=T_{s}\left(r_{0} / r\right)^{2 / n} \quad\left(r \geq r_{0}\right) .
$$

In the inner parts of the envelope where the dust is relatively warm $(\gtrsim 200 \mathrm{~K})$ this leads to a square root dependence of the dust temperature on the radius. In the outer parts this dependence flattens somewhat because of the reduced emissivity of the dust. In models of the emission of circumstellar dust, generally $Q_{\mathrm{abs}} / Q_{\mathrm{em}}$ is chosen as the free parameter rather than $r_{0}$. This sets then the inner radius of the dust shell for a given luminosity and sublimation temperature through the energy balance (see eq. [1]).

The emergent luminosity of an optically thin envelope is given by

$$
L(\lambda)=\int 4 \pi r^{2} n_{d}(r) 4 \pi a^{2} Q(\lambda) \pi B\left(\lambda, T_{d}\right) d r,
$$

where $n_{d}(r)$ is the dust particle density at distance $r, a$ the grain size, $Q(\lambda)$ the absorption efficiency at wavelength $\lambda$, and the integral is evaluated from the inner $\left(r_{0}\right)$ to the outer boundary $\left(r_{1}\right)$. Assuming a spherically symmetric outflow with a constant velocity $v$, the emergent flux can be written as

$$
F(\lambda)=4 \pi\left(r_{0} / D\right)^{2} N_{d} \pi a^{2} Q(\lambda) \int B\left[\lambda, T_{d}(x)\right] d x,
$$


where $N_{d}$ is the dust column density from $r_{0}$ to $r_{1}\left[N_{d}=\right.$ $\left.n_{d}\left(r_{0}\right) r_{0}\right], D$ is the distance, $x$ is equal to $r / r_{0}$, and the integral is calculated from 1 to $r_{1} / r_{0}$. For all practical purposes in the near- and mid-infrared the latter can be set equal to infinity, since $r_{1}$ is much larger than $r_{0}$ and the integrand is a steep function of $T_{d}$. Note that $r_{0} / D$ is just proportional to the stellar luminosity. Equation (4) can also be written as

$$
F(\lambda)=\left(3 \dot{M}_{d} / 4 v \rho_{s}\right)[Q(\lambda) / a]\left(r_{0} / D^{2}\right) \int B\left[\lambda, T_{d}(x)\right] d x,
$$

where $\dot{M}_{d}$ is the dust mass-loss rate, and $\rho_{s}$ the specific density of the dust material. In the Rayleigh limit $(2 \pi a / \lambda \lesssim 0.5) Q / a$ is independent of grain size, and the grain size does not enter into the problem. The integral can be evaluated numerically using equation (2). The results for $F(\lambda) / Q(\lambda)$ are shown in Figure 1 for two different sublimation temperatures, assuming a constant mass-loss rate and that $n$ is equal to 4 , appropriate for warm silicate dust grains. Not that $r_{0}$ scales inversely with the sublimation temperature to the $n / 2$ power (see eq. [1]) and, thus, if $F(\lambda) / Q(\lambda)$ had been evaluated for constant column density, the $T_{s}=750 \mathrm{~K}$ curve would shift upward relative to the $T_{s}=$ $1000 \mathrm{~K}$ case in Figure 1. In that case, since the optical depth through the circumstellar shell is constant, the total emitted flux and thus the area under the $F_{\lambda} / Q_{\lambda}$ curves is constant.

Several interesting aspects of this weighting integral in equation (5) can be glanced from this figure. First, in the optically thin limit all models with the same $T_{s}$ will have the same spectral shape, independent of the mass-loss rate. The absolute flux level (i.e., the contrast with the stellar infrared continuum) does depend, of course, on the mass-loss rate. Second, due to the temperature gradient, the spectra are much broader than a single blackbody (Leung 1976b). Essentially, at each wavelength this weighting integral extends to about the radius where the dust temperature provides peak emission at that the wavelength [e.g., $T_{d}(r) \approx 0.3 / \lambda \mathrm{K}$, with $\lambda$ in $\left.\mathrm{cm}\right]$. Thus at longer wavelengths a larger volume of the dust shell contributes. Third, this integral has a limit for the red end of the spectrum analogous to the Rayleigh-Jeans limit. The slope is, however, much shallower (about 3 vs. 4 for a blackbody). This is, of course, again due to the presence of cool dust far out in the shell (Leung 1976b). This should be kept in mind when deriving

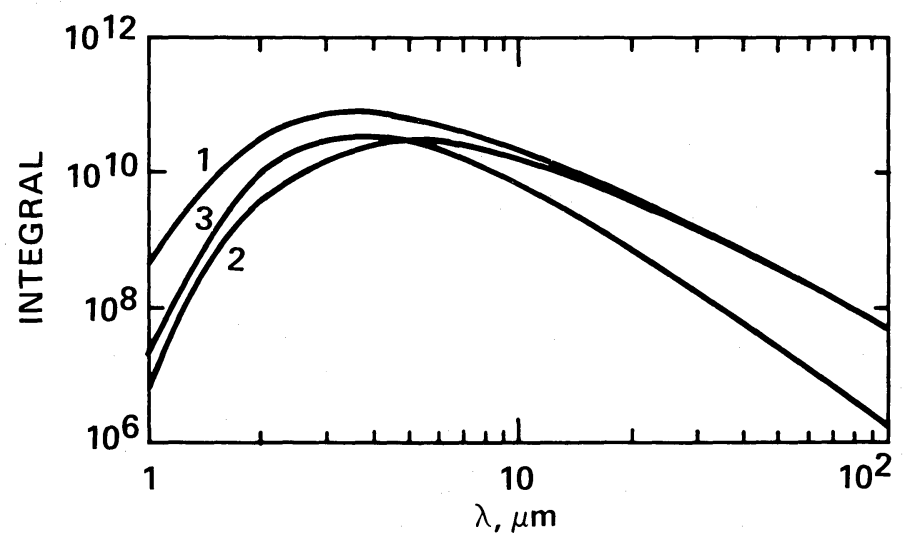

FIG. 1.-The weighting function in the analytical expression for the emergent flux (eq. [5]) for two different condensation temperatures $T_{s}=1000 \mathrm{~K}$ (1) and $T_{s}=750 \mathrm{~K}(2)$. The blackbody curve for $\mathrm{T}=1000 \mathrm{~K}$ is also shown (3). For details see text. the far-infrared absorption law for circumstellar dust from astronomical data (Martin and Rogers 1987). Conversely, when $\lambda \gg \lambda_{\max }$, where $\lambda_{\max }$ is the maximum of the Planck function at the sublimation temperature $\left(\lambda_{\max } T_{s} \approx 0.3 \mathrm{~cm} \mathrm{~K}\right)$, the temperature structure at the inner boundary is unimportant for the emergent spectrum. Fourth, if we had kept the inner radius fixed (e.g., varied $Q_{\mathrm{abs}} / Q_{\mathrm{em}}$ rather than $r_{0}$ ), the shell with the lower $T_{s}$ will have a lower average dust temperature, and the shape of the weighting integral will change somewhat. For reasonable values of $T_{s}(>750 \mathrm{~K})$, this has little effect for $\lambda>5 \mu \mathrm{m}$, except for a small scaling factor in the absolute fluxes.

Considering the shape of the $10 \mu \mathrm{m}$ emission feature in circumstellar shells, we can conclude that it is fairly insensitive to the envelope parameters in the optically thin limit and reflects rather directly the intrinsic shape of the intrinsic dust absorption feature. Of course, the low contrast with the stellar emission will severely hamper the inversion of equation (5) outside the 10 and $20 \mu \mathrm{m}$ silicate resonances for thin shells. In the optical thin limit, deriving the near-infrared absorption properties (e.g., $Q_{\mathrm{abs}} / Q_{\mathrm{em}}$ ) from the energy balance (see eq. [1]) is, obviously, also difficult as it requires knowledge on the inner radius of the circumstellar envelope.

In the optically thick limit, the radiation by the dust in the circumstellar envelope is important in heating the dust, and the temperature structure will differ from the simple optically thin limit. This makes the temperature structure a nonlocal problem and moreover couples the emission at one particular frequency to all other frequencies. This interrelationship between temperature and radiation field makes this inversion process for obtaining the absorption properties of the circumstellar dust a very complicated one requiring detailed numerical radiative transfer calculations. Because of the interwoven nature of the emission and absorption process a trial and error procedure is called for to determine the intrinsic dust absorption properties, and this will be discussed in detail in the remainder of this article. However, it should be emphasized that its main features will be quite similar to those in the optically thin limit (see Fig. 1).

\section{METHOD}

We have assumed that the mass loss from the central star is radially directed, spherically symmetric, and constant in time. A dust-free zone (radius $r_{0}$ ) with zero opacity separates the dust shell from the stellar photosphere (see $\S \mathrm{II} a$ ). The dust is assumed to condense instantaneously at $r_{0}$, and all dust grains are assumed to have the same size, $a$. The radiative transfer method is discussed in $\S$ III $a$. The dust absorption properties are discussed in $\S$ III $b$. The dust-density distribution in circumstellar shells is examined in $\S$ III $c$.

\section{a) Radiative Transfer Method}

The radiative transfer through the circumstellar dust shell is solved using the efficient "quasi-diffusion method" (Leung $1975,1976 a, b)$. This exceedingly stable and flexible numerical scheme calculates the dust temperature distribution fully consistently, taking the anisotropic nature of the radiation field into account. In principle, this method can be extended to include several grain components or sizes (Leung 1976a), although that was not considered for this application. It should be mentioned that the emergent spectrum is dependent on the ratio of the inner boundary of the dust shell to the stellar radius rather than the absolute dimensions (see eq. [1]). Like- 
wise, only the stellar effective temperature, rather than the stellar luminosity, enters into the problem (or the spectral shape when nonblackbodies are considered). Of course, once a choice for the stellar luminosity is made, it also sets the stellar radius (e.g., $L_{*}=4 \pi r_{*}^{2} \sigma T_{\text {eff }}{ }^{4}$ ) and thus the absolute dimensions of the system.

\section{b) The Dust Absorption Properties}

The absorption properties of the dust are treated independently in each of four wavelength regions: $\lambda \leq 6 \mu \mathrm{m}$, $6 \mu \mathrm{m} \leq \lambda \leq 13 \mu \mathrm{m}, \quad 13 \mu \mathrm{m} \leq \lambda \leq 25 \mu \mathrm{m}$, and $\lambda \geq 25 \mu \mathrm{m}$. Only for the first of these is scattering considered to be of importance and taken into account.

\section{i) The Near-Infrared Region}

For the short-wavelength region we have assumed that the absorption and scattering efficiencies are given by the Rayleigh limits (i.e. $2 \pi a / \lambda \leq 0.5$ ):

$$
\begin{aligned}
& Q_{\text {abs }}(\lambda)=Q_{0}[2 / \lambda(\mu \mathrm{m})] \\
& Q_{\text {sca }}(\lambda)=Q_{1}[2 / \lambda(\mu \mathrm{m})]^{4},
\end{aligned}
$$

where it is assumed that the optical constants are constant over this wavelength region. The constants $Q_{0}$ and $Q_{1}$ are always chosen in such a way as to keep the absorption and scattering cross section less than the geometrical cross section over the relevant wavelength region $(\lambda \gtrsim 1 \mu \mathrm{m})$. In all of the calculations $Q_{1}$ is fixed to $1.6 \times 10^{-4}$, while $Q_{0}$ is treated as a free parameter. For our standard model we have chosen $Q_{0}=$ 0.018 resulting in a $Q_{1} / Q_{0}$ ratio of $9 \times 10^{-3}$. For a real index of refraction of 1.7 , this choice implies a grain size of about $500 \AA$. The size distribution of interstellar grains extends to somewhat

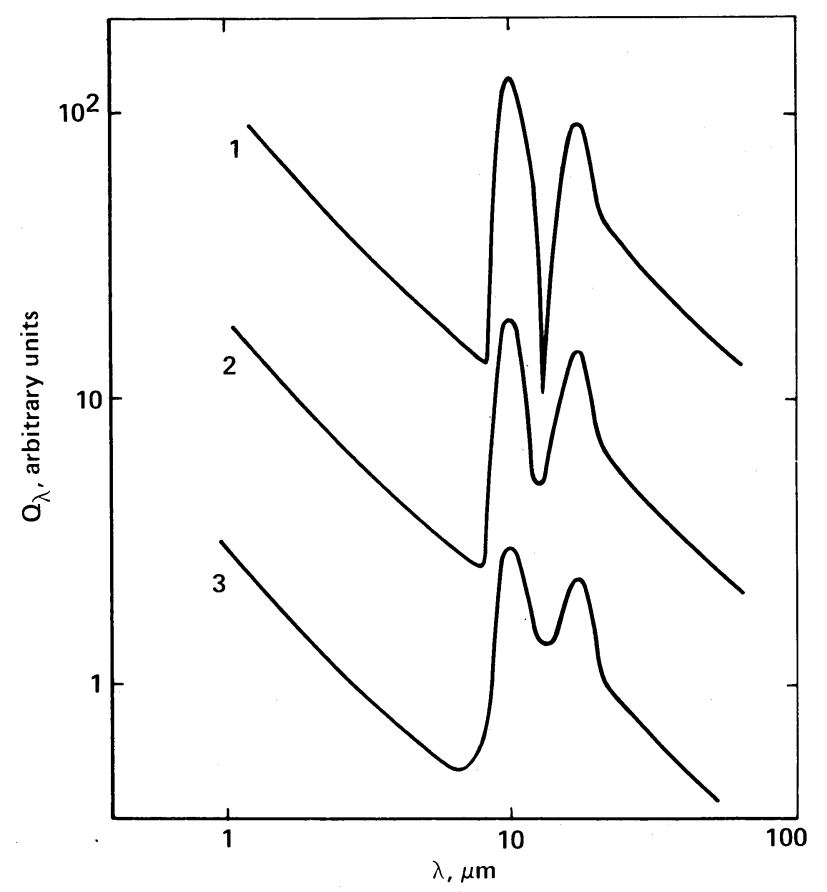

FIG. 2.-The assumed IR absorption properties for circumstellar silicates. Curves 1,2 , and 3 give the best fit to the observed 2-20 $\mu$ m spectrum of $\mathrm{OH}$ $26.5+0.6$, IRC 10011 , and R Cas, respectively. They differ mainly in the shape of the $10 \mu \mathrm{m}$ feature particularly around 8 and $13 \mu \mathrm{m}$. The shape of the $10 \mu \mathrm{m}$ feature derived for the dust in Orion (Gillett et al. 1975) is quite similar to that of curve 3 . larger sizes ( $a \lesssim 2500 \AA$; Mathis, Rumpl, and Nordsieck 1977), and departures from the Rayleigh limit might be expected. Moreover, for such large grains scattering would be very important in the near-IR $(\lambda \leq 3 \mu \mathrm{m})$. However, as discussed in $\S \mathrm{IV} c$ we suggest that the near-infrared absorption of circumstellar silicates is caused by electronic transitions in transition metals. The assumption of constant indices of refraction, appropriate for dielectric materials far from resonance, is then hardly justified. In principle, we could have assumed optical constants, a grain size, and shape, and calculated the efficiencies using Mie scattering theory (van de Hulst 1957). However, we feel that such a detailed treatment has to await actual identification of the near-infrared absorption process in circumstellar silicates. Furthermore, because of the absence of independent information on the grain size and shape (or their distribution functions), such a treatment would still contain (at least) two free parameters and a simple parametrization was felt to be more appropriate.

\section{ii) The $10 \mu \mathrm{m}$ Feature}

The $10 \mu \mathrm{m}$ range is dominated by the $\mathrm{Si}-\mathrm{O}$ stretching vibrations. Preliminary modeling of the observed infrared spectra of R Cas, IRC 10011, and OH 26.5+0.6 led to the adoption of three different shapes for this absorption feature (Fig. 2). These dust models differ only in the shape of the $10 \mu \mathrm{m}$ silicate feature, particularly around 8 and $13 \mu \mathrm{m}$. Dust model 1 has a seemingly sharper $10 \mu \mathrm{m}$ feature than model 3 . It should be emphasized, however, that the full width at half-maximum does not change very much from one model to another.

All $10 \mu \mathrm{m}$ features are normalized to the same peak absorption efficiency, $Q_{\text {peak }}$. In terms of the peak absorption strength, $S(10 \mu \mathrm{m})$, the peak absorption efficiency is given by

$$
Q_{\text {peak }}=4 a \rho_{s} S(10 \mu \mathrm{m}) / 3,
$$

where $\rho_{s}$ is the specific weight of the dust material, which is typically $2.5 \mathrm{~g} \mathrm{~cm}^{-3}$ for amorphous silicate materials. Assuming that $S(10 \mu \mathrm{m})$ is equal to $2 \times 10^{3} \mathrm{~cm}^{2} \mathrm{~g}^{-1}$, the experimentally determined value for amorphous magnesium silicates (Day 1979), and $a$ is $500 \AA$ yields $3.3 \times 10^{-2}$ for $Q_{\text {peak }}$. Note that the temperature of the warm dust and therefore the emergent near-IR spectrum depends only on the ratios of $Q_{0}$ to $Q_{\text {peak }}$ and not on the absolute values (see $\S$ II $b$ ). The assumed $10 \mu \mathrm{m}$ absorption strength will however influence the conversion from $10 \mu \mathrm{m}$ optical depth of the circumstellar shell to a total mass-loss rate.

iii) The $20 \mu \mathrm{m}$ Feature

In the $20 \mu \mathrm{m}$ region the absorption properties are dominated by the $\mathrm{Si}-\mathrm{O}$ bending vibrations. In our synthetic dust particle the $\mathrm{SiO}$ bending modes have been represented by a Lorentz oscillator which peaks at $17.5 \mu \mathrm{m}$, has a full width at half-maximum of $5.5 \mu \mathrm{m}$, and a peak strength equal to 0.65 times that of the $10 \mu \mathrm{m}$ feature. The $20 \mu \mathrm{m}$ feature has been smoothly joined to the $10 \mu \mathrm{m}$ feature at $13 \mu \mathrm{m}$. We note that because no attempt was made to reproduce in detail $20 \mu \mathrm{m}$ spectroscopic observations of Miras, the detail of the shape and strength of $20 \mu \mathrm{m}$ feature are of little importance. Once the dust emission is dominated by the $20 \mu \mathrm{m}$ feature (i.e., $T_{d} \lesssim$ $150 \mathrm{~K}$ ), the $20 \mu \mathrm{m}$ integrated strength is, of course, important in the determination of the dust temperature. The assumptions listed above are in reasonable agreement with airborne observations of circumstellar and interstellar silicates (McCarthy et al. 1980; Forrest, McCarthy, and Houck 1979). Recent IRAS 
studies suggest that the ratio of the 20 to $10 \mu \mathrm{m}$ feature lies in the range 0.35 to 0.8 (Gal et al. 1987; Bedijn 1987; Volk and Kwok 1988; Simpson and Rubin 1988). This ratio may actually vary from source to source (Volk and Kwok 1988; Bedijn 1987), and our choice should be viewed as an average value.

$$
\text { iv) The Far-Infrared Region }
$$

For a wavelength longward of $25 \mu \mathrm{m}$, the absorption efficiency is assumed to be given by

$$
Q_{\mathrm{abs}}(\lambda)=Q_{2}(25 / \lambda)^{\mathrm{n}},
$$

where $Q_{2}$ and $n$ are constants. The parameter $Q_{2}$ is chosen in such a way as to smoothly join the far-infrared opacity with the $20 \mu \mathrm{m}$ feature (i.e., $Q_{2}=0.26 Q_{\text {peak }}$ ). The far-infrared absorption efficiency of interstellar grains seems to show a $\lambda^{-1}$ wavelength dependence (Gatley et al. 1977; Campbell et al. 1976; Thronson and Harper 1979). Such a wavelength dependence is expected for layered amorphous materials, such as layer-lattice silicates. Crystalline and three-dimensional amorphous materials will give rise to a $\lambda^{-2}$ dependence in the far-infrared (Tielens and Allamandola 1987a; Tielens 1989). In the calculations $n$ is set equal to 1 . In a future publication we intend to study this assumption further. Here we merely notice that this assumption will mainly influence the temperature of grains below about $100 \mathrm{~K}$, and the exact wavelength dependence is therefore of little importance for the determination of the $1-15 \mu \mathrm{m}$ optical properties of circumstellar silicates.

The real and imaginary parts of the index of refraction of a material are related to each other through the KramersKronig relationship (e.g., causality; Wooten 1972). In principle, this relation can be used to constrain the absorption and scattering efficiencies of a material (Draine and Lee 1986). However, these efficiencies depend also on the size and shape distribution of the grains. As there is no unique relationship between optical properties and absorption and scattering efficiencies, we have chosen to ignore this additional constraint.

\section{c) The Density Distribution}

Previous studies of radiative transfer in circumstellar dust shells have assumed simple power laws for the dust density distribution (e.g., $n_{d}(r) \sim r^{-n}$ with $n=1,2$; e.g., Jones and Merrill 1976). Such a density law with $n=2$ is expected for a constant velocity and constant mass-loss stellar wind. However, close to the inner boundary the dust will be rapidly accelerated, and substantial deviations from this density distribution are expected. A correct derivation of the density distribution of the dust involves solving the coupled equations of radiative transfer and dust and gas dynamics (e.g. Martin and Rogers 1987). Following this extensive approach is beyond the scope of this paper which is aimed at correctly treating the effects of radiative transfer in deriving the parameters of the circumstellar dust shells. In deriving the density distribution function we therefore have followed a simplified approach which however preserves the essential characteristics of the circumstellar outflow. Using the simplified treatment, we derive an analytical expression for the density distribution. Subsequently, we will assess the validity of this result and compare it to more realistic models.

We assume that the grains condensate instantaneously at a certain distance from the star $r_{0}$. All grains are assumed to have the same radius $a$. The dust is momentum-coupled to the gas (Gilman 1972; Kwok 1975; Tielens 1983); that is, there are enough collisions of gas atoms with a dust particle to transfer essentially all the momentum which the particle gains from the radiation field to the colliding gas atoms. Describing the density distribution we need the equation of continuity for the gas

$$
\dot{M}=4 \pi r^{2} \rho v
$$

and for the dust

$$
\dot{M}_{d}=4 \pi r^{2} n_{d} m_{d} v_{d},
$$

where $r$ is the radial coordinate; $\dot{M}, \rho$, and $v$ are the mass-loss rate, the density, and velocity of the gas; and $\dot{M}_{d}, m_{d}, n_{d}$, and $v_{d}$ are the mass-loss rate, mass, number density, and velocity of the grains. In the formulation of the momentum equation of the gas the pressure term will be neglected for simplicity although that is clearly incorrect (Tielens 1983). The momentum equation of the gas is then given by

$$
v(d v / d r)=\left(G M_{*} / r^{2}\right)(\Gamma-1),
$$

where $M_{*}$ is the stellar mass, $G$ is the gravitational constant, and $\Gamma$ is the ratio of the drag force to the gravitational attraction. The dust and gas being momentum-coupled, the drag force equals the radiation pressure force, and $\Gamma$ is given by (Tielens 1983)

$$
\Gamma=\left(3 Q_{r p} / 4 a \rho_{s}\right)\left(L_{*} \delta / 4 \pi c G M_{*}\right)\left(v / v_{d}\right),
$$

where $Q_{r p}$ is the flux-weighted mean of the radiation pressure efficiency of the grains, $\rho_{s}$ the specific density of the grain material, $L_{*}$ the stellar luminosity, and $\delta$ the dust-to-gas mass ratio. It is assumed that the ratio of grain to gas velocity is constant. This assumption is correct in the optically thick limit but gives even for a mass-loss rate as small as $2 \times 10^{-7} M_{\odot}$ $\mathrm{yr}^{-1}$, an error of less than a factor of 2 (Tielens 1983). Neglecting scattering, $Q_{r p}$ is equal to the absorption efficiency averaged over the local flux distribution and, thus, depends on the position in the shell. For optically thin shells, $Q_{r p}$ reduces to $Q_{\text {abs }}$ averaged over the stellar flux. In the optically thick limit, photons can escape the envelope by diffusing to longer wavelength where the absorption efficiency is smaller. Thus, $Q_{r p}$ might decrease with distance from the star (i.e., temperature of the radiation field). As a first approximation we will assume that $Q_{r p}$ is equal to the optically thin value throughout the envelope. Then $\Gamma$ is constant through the flow. The importance of this assumption will be assessed in $\S \mathrm{V} c$. Finally, note that in the Rayleigh limit, $Q_{\text {abs }}$ is proportional to $a$ and thus $\Gamma$ does not depend on the grain size.

Integrating the right-hand term of equation (12) over the distance yields the gas velocity $v$ as a function of the radial coordinate $r$ :

$$
v=v_{\infty}\left[\left(x-y_{0}\right) / x\right]^{1 / 2} .
$$

Here $x$ equals $r / r_{0}, v_{\infty}$ is the limit of gas velocity at infinite distance and $y_{0}$ is given by

$$
y_{0}=1-\left(v_{0} / v_{\infty}\right)^{2},
$$

where $v_{0}$ is the outflow velocity of the gas at the condensation radius (approximately equal to the sound velocity). Due to the geometrical dilution of the radiation field, the terminal velocity of the gas in the infinite distance limit reaches a finite value. Assuming that $v_{\infty}$ is much greater than $v_{0}$ the terminal velocity 
is given by (Jura 1984)

$$
v_{\infty}=\left[2 G M_{*}(\Gamma-1) / r_{0}\right]^{1 / 2} .
$$

For typical values for Miras; $Q_{\text {abs }} / a=2500 \mathrm{~cm}^{-1}, \rho_{s}=2.5 \mathrm{~g}$ $\mathrm{cm}^{-3}, \delta=4 \times 10^{-3}, L_{*}=10^{4} L_{\odot}, M_{*}=M_{\odot}, r_{0}=2 \times 10^{14}$ $\mathrm{cm}$, and $v / v_{d}=1$, in equations (13) and (16) yield $v_{\infty} \approx 13 \mathrm{~km}$ $\mathrm{s}^{-1}$, within the range of $5-20 \mathrm{~km} \mathrm{~s}^{-1}$ for the $\mathrm{OH}$ and $\mathrm{CO}$ terminal velocities typically observed for Miras. We are mainly interested in modeling near- and mid-infrared spectra, and the outer radius of the envelope in our models is typically about $10^{16} \mathrm{~cm}$. Beyond this radius the envelope does not contribute appreciably to the emission or the optical depth.

Using mass conservation (equations [10] and [11]) and equation (14) yields

$$
n_{d}=n_{0} /\left[\left(x-y_{0}\right)^{1 / 2} x^{3 / 2}\right]
$$

where $n_{0}$ is given by

$$
n_{0}=\dot{M} \delta /\left(4 \pi r_{0}{ }^{2} v_{\infty} m_{d}\right)\left(v / v_{d}\right) .
$$

In the calculations, $v / v_{d}$ is assumed to be constant. At large distances this equation gives an $r^{-2}$ dependence for the density, consistent with the grains reaching a finite terminal velocity. When $y_{0}$ is equal to 0 we recover the expected $r^{-2}$ density law throughout the whole flow, and $n_{0}$ is the density at the inner radius. However, typically, $y_{0}$ is close to 1 and the density distribution falls steeply away from the inner boundary due to the acceleration by the radiation force. The steepness of the density distribution is governed by the value of $y_{0}$. This is illustrated in Figure 3. In this figure we compare the simple analytical result with the more elaborate numerical calculation for an optically thin envelope by Tielens (1983). It turns out that the differences between our analytical approximation and the more elaborate model calculation are small for $y_{0}$ around 0.96 . The influence of the density distribution on the emitted IR spectrum will be studied by varying $y_{0}$.

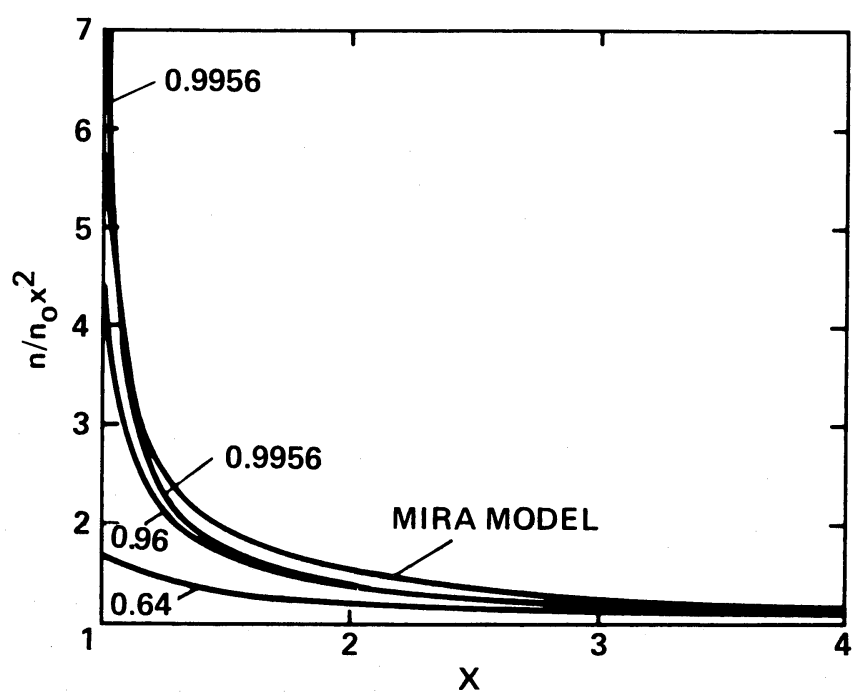

Fig. 3.-The dust density in the radiative acceleration zone is shown as a function of $x=r / r_{0}$, as given by the analytical expression [17]. The density has been divided by $n_{0}$ and multiplied by $\left(r / r_{0}\right)^{2}$ to emphasize the convergence toward the results for a constant outflow velocity. The curves are labeled by the values of $y_{0}$. The results of a detailed numerical calculation (Tielens 1983) are also shown.
The total dust column density, $N_{d}$, through the circumstellar shell can be found by integrating equation (17) from $x$ is 1 to $r_{1} / r_{0}$. This yields

$$
N_{d}=2 n_{0} r_{0}\left[1-\left(1-y_{0}\right)^{1 / 2}\right] / y_{0} .
$$

For $y_{0}$ is zero (i.e., constant outflow velocity), the column density is equal to $n_{0} r_{0}$. When $y_{0}$ is larger than zero, the dust piles up at the inner boundary, and the column density will be larger than in the constant outflow case. In the limit of $y_{0}$ goes to one, the dust column density is twice that of the constant outflow case and, thus, an appreciably larger column density can build up in the dust acceleration zone. For later use, we derive the gas mass-loss rate in terms of the $10 \mu \mathrm{m}$ optical depth, $\tau(10 \mu \mathrm{m})$, using equations (10), (11), and (19):

$$
\begin{aligned}
\dot{M}= & 2 \pi r_{0} v_{\infty}[\tau(10 \mu \mathrm{m}) / S(10 \mu \mathrm{m})]\left(v_{d} / v\right) \\
& \times\left\{y_{0} /\left[1-\left(1-y_{0}\right)^{1 / 2}\right]\right\} / \delta,
\end{aligned}
$$

where $S(10 \mu \mathrm{m})$ is the $10 \mu \mathrm{m}$ peak absorption strength. Thus, for the same $10 \mu \mathrm{m}$ optical depth, the derived mass-loss rate assuming $y_{0}=1$ is half that obtained for a constant outflow velocity.

\section{d) Free Parameters}

The free parameters in the calculation include the inner $\left(r_{0}\right)$ and outer $\left(r_{1}\right)$ radius of the dust shell. Because the emergent spectrum is sensitive to the dust temperature at the inner radius rather than the inner radius itself, generally this dust temperature (i.e., the condensation temperature $T_{s}$ ) is treated as a free parameter. Numerically, it is, however, more advantageous to choose the inner radius. For this reason the inner radius is then determined by a trial and error method in such a way that the temperature at the inner boundary is within $1 \%$ of the chosen condensation temperature of the dust. The dust density distribution is completely specified by the parameter $y_{0}$, and the $10 \mu \mathrm{m}$ dust optical depth (see equations [17]-[19]). The $10 \mu \mathrm{m}$ optical depth and the chosen inner radius determine the total mass-loss rate through equation (20). The luminosity, $L_{*}$, and the effective temperature $T_{\text {eff }}$, are also free parameters. The dust absorption and scattering properties also have to be specified. As discussed in $\S$ III $a$, these are completely described by the value of the absorption efficiency at $2 \mu \mathrm{m}, Q_{0}$, and the detailed shape of the $10 \mu \mathrm{m}$ silicate feature (i.e., model 1, 2, or 3 in Fig. 2).

Table 1 lists the values of the free parameters in our standard model. A large grid of models has been calculated. Each parameter has been varied independently. For each parameter choice, a set of models has been calculated by varying the total dust column density (i.e., the mass-loss rate). The results of these calculations are discussed in Appendix A.

\section{THE NEAR-INFRARED OPACITY OF CIRCUMSTELLAR SILICATES \\ a) The Observed $A_{10}-T_{c}$ Relation}

The ratio of the near-infrared to $10 \mu \mathrm{m}$ absorption cross section of circumstellar silicates can be determined by comparing the $2 \mu \mathrm{m}$ flux with the strength of the $10 \mu \mathrm{m}$ feature in emission or absorption (Jones and Merrill 1976; Bedijn 1977). We have compiled near-infrared data of Miras and OH/IR stars from the literature (Merrill and Stein 1976a, $b$; Evans and Beckwith 1977; Werner et al. 1980; Engels 1982; Willems and de Jong 1982; Herman 1983; Baud et al. 1985). For each 
TABLE 1

STANDARd MOdel PARAMETERs ${ }^{\mathrm{a}}$

\begin{tabular}{cc}
\hline \hline Parameter & Standard Model \\
\hline$L_{*}\left(L_{\odot}\right) \ldots \ldots \ldots \ldots$ & $10^{4}$ \\
$T_{\text {eff }}(\mathrm{K}) \ldots \ldots \ldots \ldots \ldots$ & 2000 \\
$y_{0} \ldots \ldots \ldots \ldots \ldots \ldots$ & 0.96 \\
$T_{s}(\mathrm{~K}) \ldots \ldots \ldots \ldots \ldots$ & 1000 \\
$Q_{0} / Q_{\text {peak }} \ldots \ldots \ldots \ldots$ & 0.40 \\
Dust model $\ldots \ldots$. & $1^{\mathrm{b}}$ \\
$r_{*}(\mathrm{~cm}) \ldots \ldots \ldots \ldots$. & $4.2(13)$ \\
$r_{0}(\mathrm{~cm}) \ldots \ldots \ldots \ldots$ & $1.1(14)^{\mathrm{c}}$ \\
\hline
\end{tabular}

${ }^{a}$ The dust column density (or equivalently the mass-loss rate) has been varied to construct families of models. For each of these families one of the free parameters has been varied.

${ }^{b}$ See Fig. 2.

${ }^{c}$ In the optically thin limit.

source, the color temperature, $T_{c}$, between about $3.8 \mu \mathrm{m}$ and $12.5 \mu \mathrm{m}$ has been determined from narrow-filter photometry. For some sources only $3.4 \mu \mathrm{m}$ data were available. This introduces some scatter in the derived color temperatures, mainly because the $3.4 \mu \mathrm{m}$ filter is on the edge of the photospheric $\mathrm{H}_{2} \mathrm{O}$ absorption band and consequently the $3.4 \mu \mathrm{m}$ flux may vary somewhat with stellar phase (Strecker, Erickson, and Witteborn 1978). The $12.5 \mu \mathrm{m}$ filter is on the edge of the $10 \mu \mathrm{m}$ silicate feature. Despite the problems associated with the use of these filters they are preferred over the $K$ and $M$ filters ( 2.2 and $4.8 \mu \mathrm{m}$ ), for example, because scattering may be important at the shortest wavelengths and because data are available for more objects (i.e., the OH/IR stars), and the use of an as large as possible span in $T_{c}$ is in our opinion more important than the increased observational scatter.

The strength of the $10 \mu \mathrm{m}$ feature, $A_{10}$, has been measured on a magnitude scale by assuming a linear continuum from 8.7

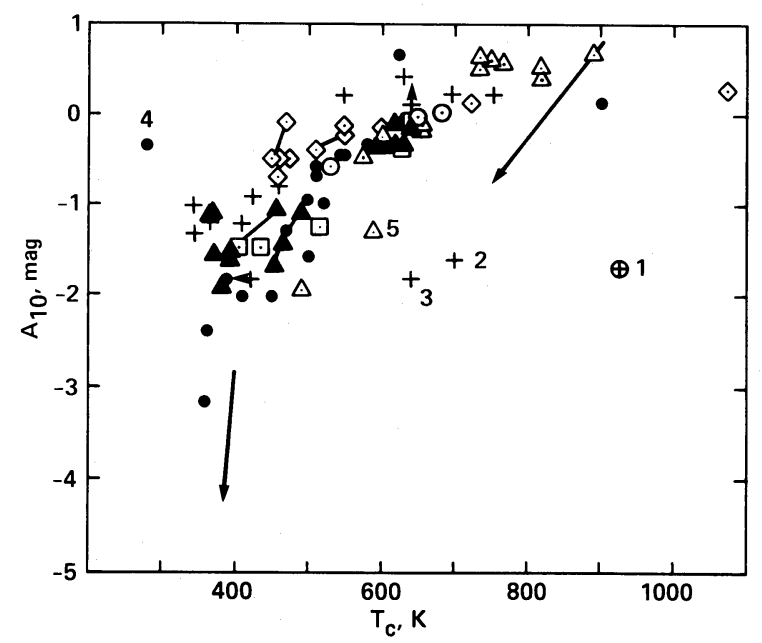

Fig. 4.-The observed relation between the strength of the $10 \mu \mathrm{m}$ feature and the 3.8-12.5 $\mu \mathrm{m}$ color temperature. Data have been taken from $(\triangle)$ Merrill and Stein $(1976 a, b) ;(\Lambda)$ Evans and Beckwith (1977); ( $\square$ ) Werner et al. (1980); $(\diamond)$ Willems and de Jong (1982); (O) Engels (1982); (๑) Herman (1983), and (+) Baud et al. (1985). Observations of an object at different phases by the same observers are connected by lines. The arrows show the effects of 30 visual magnitudes of interstellar extinction. The objects $\mathrm{OH} 0739, \mathrm{OH} 0.2+0.0, \mathrm{OH}$ 4.6-0.4, $\mathrm{OH} 17.7-2.0$, and AFGL 2885 are indicated by 1, 2, 3, 4, and 5, respectively. to $12.5 \mu \mathrm{m}$, that is

$$
A_{10}=2.5 \log \left(F_{\text {obs }} / F_{\mathrm{c}}\right),
$$

where $F_{\text {obs }}$ and $F_{c}$ are the observed and continuum flux at the depth of the $10 \mu \mathrm{m}$ feature $(9.7 \mu \mathrm{m})$. Note that in this notation positive $A_{10}$ corresponds to $10 \mu \mathrm{m}$ emission features. Generally, narrow-band photometry data have been used, except for the spectrophotometric data for some IRC and AFGL sources taken from Merrill and Stein $(1976 a, b)$. Some of the data pertain to $12.2 \mu \mathrm{m}$ rather than $12.5 \mu \mathrm{m}$ (Engels 1982; Willems and de Jong 1982). It should be emphasized that $A_{10}$ underestimates the true strength of the $10 \mu \mathrm{m}$ feature, because the $8.7 \mu \mathrm{m}$ filter and to a lesser extent the $12.5 \mu \mathrm{m}$ filter are on the wing of the $10 \mu \mathrm{m}$ feature rather than the continuum. From the shape of the interstellar $10 \mu \mathrm{m}$ extinction feature (Becklin et al. 1978), this effect is estimated to be about a factor of 2 . Furthermore, the data obtained with narrow photometric filters $(\Delta \lambda \approx 1 \mu \mathrm{m})$ also tend to underestimate the true $10 \mu \mathrm{m}$ optical depth. Of course, the presence of $10 \mu \mathrm{m}$ emission as well as absorption in the circumstellar envelope of the heavily obscured OH/IR stars makes $A_{10}$ only an indirect measure of the total dust column density anyway. One of the goals of this section is to quantify the relationship between $A_{10}$ and the dust column density or mass-loss rate.

The observational data are plotted in Figure 4. Individual observations of the same objects with the same color system are connected by lines. There is a general correlation between $A_{10}$ and $T_{c}$. Since $T_{c}$ is mainly a measure for the color excess between 3.8 and $12.5 \mu \mathrm{m}$ (e.g., $A_{3.8}-A_{12.5}$ ), especially for large dust optical depths, this correlation reflects the relation between the 3.8 and $10 \mu \mathrm{m}$ dust opacity (Kwan and Scoville 1976). Each of the individual observational data sets displays a similar $A_{10}-T_{c}$ relation, and we note that some of the apparent scatter in the combined data set may be due to the use of different color systems in these observational studies. In particular, some data pertain to $12.2 \mu \mathrm{m}$ rather than $12.5 \mu \mathrm{m}$ (Engels 1982; Willems and de Jong 1982). As a result $A_{10}$ is overestimated with respect to the data obtained at $12.5 \mu \mathrm{m}$ and these data fall consistently above the relation defined by the other observations. In the comparison with theoretical studies in the next subsection, these data are ignored.

Finally, we would like to draw attention to the variations in $A_{10}$ and $T_{c}$ with phase for individual objects. These tend to be parallel to the general correlation (Fig. 5), supporting the notion that this trend reflects intrinsic properties of the circumstellar envelopes. Clearly, the temperature structure of the envelope changes with phase. This will be discussed further below.

\section{b) Theoretical Studies}

Theoretical $A_{10}$ versus $T_{c}$ curves have been determined from the extensive model grid calculations. Some of these are compared to the observations in Figure 5. The stellar luminosity has no effect on the relationship between $A_{10}$ and $T_{c}$ (see Appendix A). The effective temperature is only important for $T_{c} \gtrsim 650 \mathrm{~K}$ or $A_{10} \gtrsim-0.3$. For the standard model this corresponds to $\tau(10 \mu \mathrm{m}) \lesssim 5$. The dust condensation temperature also influences the relation for $T_{c} \gtrsim 500 \mathrm{~K}$ or $\dot{A}_{10} \gtrsim-1$ (see Fig. 5a). This corresponds to $\tau(10 \mu \mathrm{m}) \lesssim 10$ for the standard model. Similarly, keeping the inner dust radius constant (e.g., the inner shell temperature increases with increasing column density) affects the $A_{10}-T_{c}$ relation for $\tau(10 \mu \mathrm{m}) \gtrsim 10$ (see Fig. $5 b$ ). The influence of $y_{0}$ on the $A_{10}-T_{c}$ relation is small in 

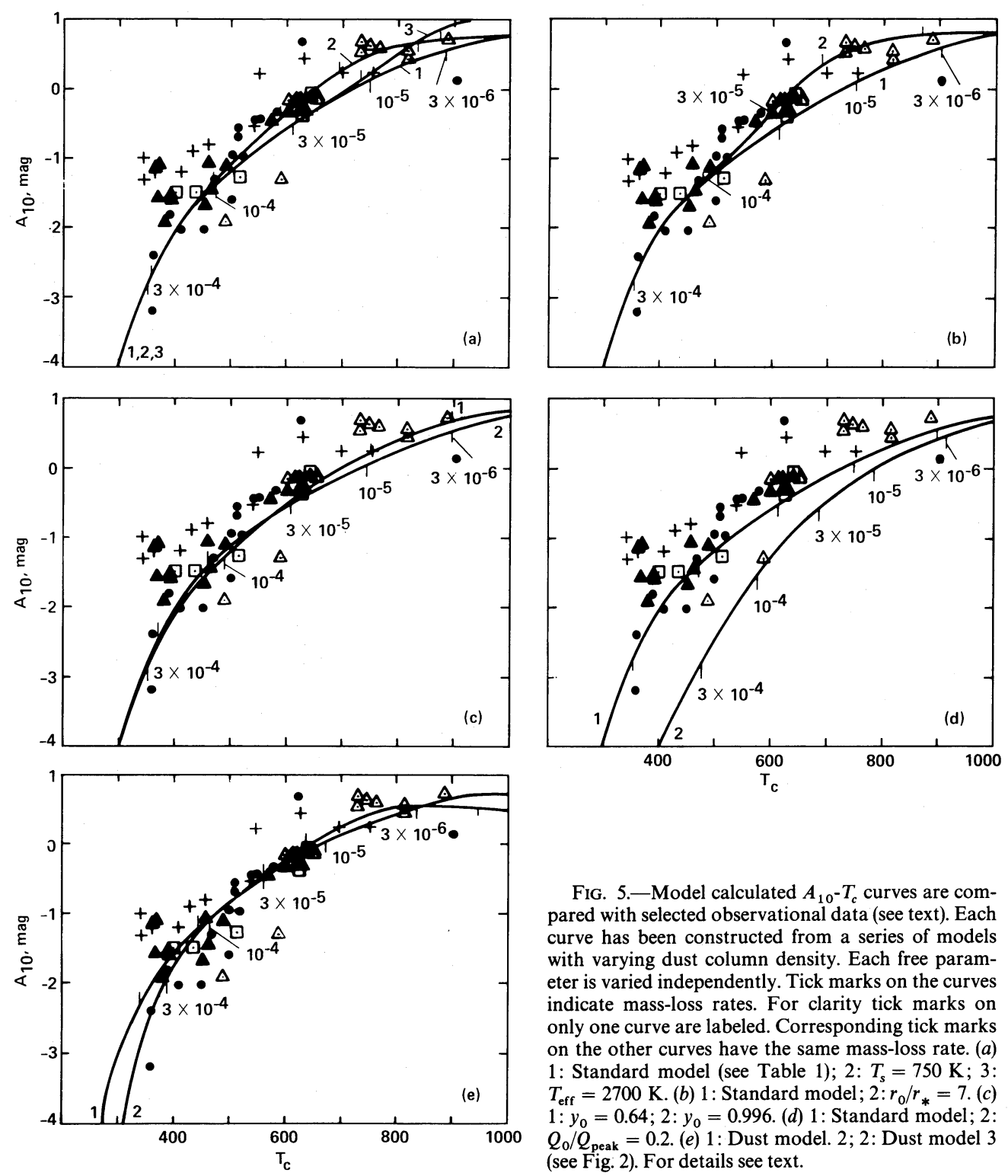

Fig. 5.- Model calculated $A_{10}-T_{\mathrm{c}}$ curves are compared with selected observational data (see text). Each curve has been constructed from a series of models with varying dust column density. Each free parameter is varied independently. Tick marks on the curves indicate mass-loss rates. For clarity tick marks on only one curve are labeled. Corresponding tick marks on the other curves have the same mass-loss rate. $(a)$ 1: Standard model (see Table 1); $2: T_{s}=750 \mathrm{~K} ; 3$ : $T_{\text {eff }}=2700 \mathrm{~K}$. (b) 1 : Standard model; $2: r_{0} / r_{*}=7$. (c) 1: $y_{0}=0.64 ; 2: y_{0}=0.996$. (d) $1:$ Standard model ; $2:$ $Q_{0} / Q_{\text {peak }}=0.2$. (e) 1 : Dust model. $2 ; 2$ : Dust model 3 (see Fig. 2). For details see text.

general (see Fig. $5 c$ ), as expected from the discussion in Appen$\operatorname{dix} A$.

Because of the dependence of the $A_{10}-T_{c}$ relation on the stellar effective temperature and dust sublimation temperature for $\tau(10 \mu \mathrm{m}) \lesssim 10$, it is difficult to determine the near-infrared dust absorption properties from Miras and IRC sources alone. For the highly optically thick $\mathrm{OH} / \mathrm{IR}$ stars the $A_{10}$ versus $T_{c}$ relation depends mainly on the near-infrared absorption properties of the dust. Essentially this is because the highly optically thick shell "muffles" the inner boundary condition (see Appendix A). This is illustrated in Figure $5 d$ which shows this relationship for two different values of the near-infrared to $10 \mu \mathrm{m}$ absorption efficiency. As expected, for the same apparent $10 \mu \mathrm{m}$ optical depth the observed spectrum is cooler for a higher ratio owing to the increased near-infrared absorption. The detailed shape of the assumed $10 \mu \mathrm{m}$ feature has also some influence on the $\mathrm{A}_{10}-T_{c}$ relationship. This is because the continuum points at 8.7 and $12.5 \mu \mathrm{m}$, used to measure $A_{10}$, are actually on the wings of the $10 \mu \mathrm{m}$ feature. This is illustrated in
Figure $5 e$ for dust models 2 and 3 (see Fig. 2). The different model curves in Figure $5 e$ are intertwined due to complicated radiative transfer effects (i.e., emission and [self]-absorption in the $10 \mu \mathrm{m}$ feature). In any case the differences are small compared to those introduced by the variation of the near-infrared absorption efficiency. As will be discussed in $\S \mathrm{V}$ the shape of the $10 \mu \mathrm{m}$ feature in the standard model gives a good fit to the spectrum of the $\mathrm{OH} / \mathrm{IR}$ star $\mathrm{OH} 26.5+0.6$. We will assume that this shape is a general characteristic of circumstellar silicates in such highly obscured stars. The ratio of the $2 \mu \mathrm{m}$ to $10 \mu \mathrm{m}$ absorption efficiency, $Q_{0} / Q_{\text {peak }}$, may be slightly higher $(\sim 0.5)$ than assumed in our standard model calculations. The observational scatter precludes, however, a better determination of this parameter. We note that this value gives a good fit to the observed spectrum of $\mathrm{OH} 26.5+0.6(\mathrm{see} \S \mathrm{V} c)$. The standard model falls consistently below the data for $T_{c} \gtrsim$ $600 \mathrm{~K}$. Inspecting Figure 5 suggests that this is due to a lower dust condensation temperature $\left(T_{s} \approx 750 \mathrm{~K}\right)$ in these circumstellar envelopes. Indeed, some detailed studies have suggested 
that a dust condensation temperature of $500 \mathrm{~K}$ might provide a better fit to the IR observations of many optically thin dust shells (Rowan-Robinson and Harris 1982). The difference between this $T_{s}$ and that derived from Figure 5 results from a difference in $Q_{0} / Q_{\text {peak }}$ (see below).

The derived value for $Q_{0} / Q_{\text {peak }}$ is comparable to that derived by Bedijn $(0.36 ; 1977)$ and a factor of 2 less than that of Jones and Merrill $(0.8 ; 1976)$. These values were obtained by determining the dust column density for which the $10 \mu \mathrm{m}$ feature goes into self-absorption and comparing the near-infrared flux with that expected from the obscured star. Typically, selfabsorption occurs around $T_{c} \approx 600 \mathrm{~K}$ (i.e., IRC sources). In this regime the observed spectra are more sensitive to other parameters than the spectra of $\mathrm{OH} / \mathrm{IR}$ sources (see Appendix A). Our calculations show that a value of 0.8 for $Q_{0} / Q_{\text {peak }}$ (Jones and Merrill 1976) is excluded for the heavily obscured $\mathrm{OH} / \mathrm{IR}$ stars. For more optically thin envelopes (i.e., $\tau[10 \mu \mathrm{m}] \lesssim 10$ ), such a high value is allowed if the dust condensation temperature is simultaneously lowered to about $500 \mathrm{~K}$ (see Fig. 5a; Rowan-Robinson and Harris 1982). However, this implies that dust condensation takes place at extremely large distances above the stellar photosphere $\left(r_{0} \approx\right.$ $\left.5 \times 10^{15}-10^{16} \mathrm{~cm} \approx 100 r_{*}\right)$. This is much larger than commonly observed (see Beckwith 1985). Moreover, it would be difficult to transport photospheric material to such large distances. Thus, we conclude that the ratio of the near-infrared to $10 \mu \mathrm{m}$ absorption efficiency is fairly independent of the shell optical depth $\left(Q_{0} / Q_{\text {peak }} \approx 0.5\right)$. Small variation $(\sim 25 \%)$ may, however, be present (see $\S \mathrm{V})$.

\section{c) The Near-Infrared Absorption of Silicates}

The derived intrinsic, near-infrared (1-5 $\mu \mathrm{m})$ absorption of circumstellar silicates is much higher than for common terrestrial materials, presumably due to the presence of impurities (Jones and Merrill 1976; Bedijn 1977). For this reason interstellar silicates are often nicknamed "dirty" silicates. Quantitatively, our analysis of a large body of observations indicates that the absorption at $1 \mu \mathrm{m}$ is about comparable to that at the peak of the $10 \mu \mathrm{m}$ absorption feature. The origin of this high absorptivity is not understood. In principle, three fundamentally different processes could be responsible for this high absorptivity: (1) fundamental vibrations, (2) a separate dust component, and (3) electronic transitions in impurities.

The fundamental vibrations of silicate materials occur around 10 and $20 \mu \mathrm{m}$. Overtones and combination bands of these vibrations, which do occur throughout the 1-5 $\mu \mathrm{m}$ region, are too weak relative to the fundamentals to play a role in the enhanced near-infrared absorptivity of circumstellar silicates. Water of hydration has a relatively strong absorption feature around $3 \mu \mathrm{m}$, but it is generally very narrow and covers only a small fraction of the stellar spectrum. Its importance is further reduced by strong absorption by gaseous $\mathrm{H}_{2} \mathrm{O}$ in the photosphere. Thus fundamental vibrations cannot be responsible for the high near-IR absorptivity of circumstellar silicates.

Free electrons in metallic iron particles-either as a separate dust component (Taam and Schwartz 1976) or embedded in the silicate material - may give rise to additional absorption in the near-IR. Following Greenberg (1968), it can be estimated that metallic iron particles embedded in the silicate grains may give rise to an imaginary part of the index of refraction of at most $4 \times 10^{-3}$ around $1 \mu \mathrm{m}$. This is much less than the required 0.07 , essentially because the wavelength of interest is very far from the plasma frequency. Likewise, radiative transfer studies require about 10 times more $\mathrm{Fe}$ in iron grains than $\mathrm{Si}$ in "clean" silicate grains to produce a good fit to the observed spectrum of the late-type giant VY CMa (Taam and Schwartz 1976). Furthermore, thermodynamic equilibrium calculations show that metallic iron grains are not expected to form in the outflow unless the pressure is at least $10^{-4}$ atmosphere (Kozasa and Hasegawa 1988). This is four to six orders of magnitude larger than the expected pressures in the dust condensation zones of oxygen-rich giants. In fact, under these conditions most of the iron is expected to be incorporated as $\mathrm{FeO}$ into $\mathrm{Mg}$ silicates (i.e., $(\mathrm{Fe}, \mathrm{Mg}) \mathrm{SiO}_{3}$ or $\left.(\mathrm{Fe}, \mathrm{Mg})_{2} \mathrm{SiO}_{4}\right)$. This problem is further compounded by the highly supercooled conditions, which will dominate the dust-formation process. As a result, dust condensation is expected to be very heterogeneous, and formation of more than one separate dust component is highly unlikely. Thus, metallic iron grains are not a viable source for the high near-IR absorptivity in circumstellar shells.

Electronic transitions in color centers form a third possibility for the high near-infrared absorptivity of circumstellar silicates. Dissolved transition (or rare earth) metals give rise to the multitude of colors employed in many artistic and functional applications of glasses (Bates 1962; Wong and Angell 1976). The $3 d$ electronic levels of such elements, which are degenerate in the gaseous state, will be split by the nonuniform electric field exerted by neighboring anions (i.e., ligands). Although transitions between these levels are formally forbidden by selection rules, mixing of the $3 d$ and metal $4 p$ orbitals makes them weakly allowed. The energy splitting between the $3 d$ levels depends on the particular valence state of the transition metal as well as the geometry, nature, and coordination of the ligands. A survey of the extensive literature (Bamford 1962; Schulman and Compton 1962; Wong and Angell 1976; Fanderlik 1983) on the colors of transition metaldoped glasses shows that only dissolved ferrous iron (i.e., $\mathrm{Fe}^{2+}$ ) shows appreciable near-infrared absorptivity, ranging from about $7000 \AA$ to $2 \mu \mathrm{m}$ and centered at about $1 \mu \mathrm{m}$. However, in soda-lime glass containing traces of iron (e.g., $3 \mathrm{Na}_{2} \mathrm{O} .7 \mathrm{SiO}_{2}$ containing $0.5 \mathrm{wt} \%$ of $\mathrm{Fe}$ ) the peak strength of this absorption feature is measured to be only $301 \mathrm{~mole}^{-1} \mathrm{~cm}^{-1}$ (Steele and Douglass 1965; Edwards, Paul, and Douglas 1972; Bishay and Kinawi 1965). This corresponds to a peak absorption coefficient of $5 \times 10^{-20} \mathrm{~cm}^{2}$ per $\mathrm{Fe}^{2+}$ ion, which is about a factor of 10 less than the peak absorptivity of the $\mathrm{SiO}$ stretch in amorphous silicates (Tielens and Allamandola 1987a). This forms a serious problem for this possibility. However, the strength of this electronic transition will depend on the presence of other cations in the silicate network as well as the (lack of) symmetry around the dissolved metal ion. Indeed, this feature seems much more prominent in phosphate glasses when $\mathrm{Na}$ is replaced by $\mathrm{Mg}$ (Wong and Angell 1976). Likewise peridot, a Mg-silicate containing about $10 \mathrm{wt} \%$ of $\mathrm{Fe}$, seems to show a relatively strong $1 \mu \mathrm{m} \mathrm{Fe}^{2+}$ absorption (Farrell and Newnham 1965). In fact, the characteristic green color of this mineral is probably caused by the tail of this absorption band extending into the red. A similar large dependence of the strength of the $\mathrm{Fe}^{2+}$ absorption band on composition is indicated by experimental studies of borate glasses (Bishay and Kinawi 1965; Farrell and Newnham 1965). It is therefore perhaps possible that the high observed absorptivity of circumstellar silicates is due to electronic transitions in dissolved transition metals. Further laboratory studies of more relevant silicate materials are imperative to confirm this possibility. 


\section{d) The Mass-Loss Rate}

The mass-loss rate as a function of the dust column density (i.e., optical depth) is given by equation (20). We have calculated the mass-loss rate for our theoretical model calculations using $v_{d} / v=1, v_{\infty}=10 \mathrm{~km} \mathrm{~s}^{-1}, \delta=4 \times 10^{-3}, S(10 \mu \mathrm{m})=$ $2 \times 10^{3} \mathrm{~cm}^{2} \mathrm{~g}^{-1}$, and $L_{*}=10^{4} L_{\odot}$. Tick marks along the theoretical curves in Figure 5 give the mass-loss rate at regular intervals. Since the position on the $A_{10}-T_{c}$ locus is directly proportional to the dust column density for the optically thick shells, the derived mass-loss rates are expected to be fairly independent of the choice of free parameters. This is borne out by a close examination of Figure $5 a-e$. Direct determination of dust column densities and mass-loss rates from the observed depth of the $10 \mu \mathrm{m}$ feature is hampered by complicated radiative transfer effects, and emission as well as absorption in the $10 \mu \mathrm{m}$ feature has to be taken into account. Similarly, direct determination from the observed color temperature requires knowledge on the near-infrared absorption efficiency of the dust. Essentially, the $A_{10}-T_{c}$ relation calibrates this efficiency in terms of the $10 \mu \mathrm{m}$ absorption efficiency. Note that the derived $Q_{0} / Q_{\text {peak }}$ also directly enters into the determination of the radius of the inner boundary, the other parameter required to convert optical depth into mass-loss rate (i.e., the energy balance; eq. [1]). Besides $Q_{0} / Q_{\text {peak }}$, the energy balance also depends on the assumed values for the condensation temperature of the dust, $T_{s}$, and the assumed luminosity of the star. The insensitivity of the $A_{10}-T_{c}$ relation to $T_{s}$ for large dust column densities (see Fig. $5 a$ ) should perhaps be commented upon. The overall drop in dust temperature through the envelope with decreasing condensation temperature results in a decrease in the column density required to fit a given color temperature (or $A_{10}$; see Appendix A $d$ ). However, in calculating the corresponding mass-loss rate this is largely offset by the resulting increase in the radius of the inner boundary. Note that for optically thin envelopes mass-loss rates derived from the strength of the $10 \mu \mathrm{m}$ feature in emission do depend on the assumed condensation temperature (see Fig. 5a and eq. [5]). Finally, although the luminosity of the star is important only for the absolute scale of the emitted spectrum and not for such relative measures as optical depths, it does influence the massloss rate through the absolute value of the inner boundary (i.e., $\dot{M} \sim L_{*}{ }^{1 / 2}$; see eq. [1]).

The insensitivity of the $A_{10}-T_{c}$ relation to the details of the radiative transfer makes it a prime candidate for the determination of mass-loss rates from oxygen-rich giants. Examination of Figure 5 shows that optically visible Mira variables typically have mass-loss rates less than $10^{-5} M_{\odot} \mathrm{yr}^{-1}$, while the IRC sources range from $3 \times 10^{-6}$ to $3 \times 10^{-5} M_{\odot} \mathrm{yr}^{-1}$, and the $\mathrm{OH} / \mathrm{IR}$ stars from $3 \times 10^{-5}$ to $3 \times 10^{-4} M_{\odot} \mathrm{yr}^{-1}$. The maximum mass-loss rate observed is about $10^{-3} M_{\odot}$ $\mathrm{yr}^{-1}$, although the presence of foreground extinction might affect this estimate considerably (see Fig. 4 and below). Individual mass-loss rates for the objects shown in Figure 5 are listed in Table 2. These mass-loss rates are uncertain by a factor of $\sim 3$ due to possible source-to-source variations in such parameters as $L_{*}$ and $v_{\infty}$. Slightly larger uncertainties are associated with optically thin shells due to possible variations in $T_{\text {eff }}, T_{s}$, and the dust distribution at the inner boundary. Finally, as shown in Figure 4, spectral variations as a function of phase of these variable stars moves them generally parallel to the $A_{10}-T_{c}$ relation. This is mainly due to luminosity variations.

Some further uncertainty in the derived mass-loss results
TABLE 2

Mass-Loss Rates Derived from the $A_{10}-T_{c}$ Relation

\begin{tabular}{|c|c|c|}
\hline ОвЈеCт & $\begin{array}{c}\text { Mass-Loss RATES }\left(M_{\odot} / \mathrm{yr}\right) \\
\text { IR }\end{array}$ & $\mathrm{CO}$ \\
\hline RS Vir . . . . & $4.00 \mathrm{E}-06$ & \\
\hline WX Ser ............. & $1.50 \mathrm{E}-05$ & \\
\hline GL $1686 \ldots \ldots \ldots \ldots$ & $7.00 \mathrm{E}-06$ & \\
\hline GL $2885 \ldots \ldots \ldots \ldots$ & $6.00 \mathrm{E}-05$ & \\
\hline IRC $00102 \ldots \ldots \ldots$ & $7.00 \mathrm{E}-06$ & \\
\hline IRC $10011 \ldots \ldots \ldots$ & $1.90 \mathrm{E}-05$ & $2.60 \mathrm{E}-05$ \\
\hline IRC $20281 \ldots \ldots \ldots$ & $8.00 \mathrm{E}-06$ & \\
\hline IRC $30187 \ldots \ldots \ldots$ & $6.00 \mathrm{E}-06$ & \\
\hline IRC $40149 \ldots \ldots \ldots$ & $6.00 \mathrm{E}-06$ & $1.60 \mathrm{E}-05$ \\
\hline IRC $40156 \ldots \ldots \ldots$ & $8.00 \mathrm{E}-06$ & \\
\hline IRC $40448 \ldots \ldots \ldots$ & $2.80 \mathrm{E}-05$ & \\
\hline IRC $50137 \ldots \ldots \ldots$ & $1.90 \mathrm{E}-05$ & $6.50 \mathrm{E}-06$ \\
\hline IRC $60150 \ldots \ldots \ldots$ & $3.00 \mathrm{E}-06$ & $3.70 \mathrm{E}-06$ \\
\hline OH $12.8-1.9 \ldots \ldots$. & $3.00 \mathrm{E}-05$ & \\
\hline OH $20.2-0.1 \ldots \ldots$. & $5.50 \mathrm{E}-05$ & \\
\hline OH $21.5+0.5 \ldots \ldots$. & $2.00 \mathrm{E}-04$ & \\
\hline OH $26.2-0.6 \ldots \ldots$. & $1.00 \mathrm{E}-04$ & \\
\hline OH $26.4-1.9 \ldots \ldots$. & $4.00 \mathrm{E}-05$ & \\
\hline OH $26.5+0.6 \ldots \ldots$. & $1.00 \mathrm{E}-04$ & $5.00 \mathrm{E}-07$ \\
\hline OH $28.7-0.6 \ldots \ldots$. & $4.00 \mathrm{E}-05$ & \\
\hline OH $30.1-0.2 \ldots \ldots$. & $3.00 \mathrm{E}-05$ & \\
\hline $\mathrm{OH} 30.1-0.7 \ldots \ldots$. & $1.50 \mathrm{E}-04$ & \\
\hline OH $30.7+0.4 \ldots \ldots$. & $1.00 \mathrm{E}-04$ & \\
\hline $\mathrm{OH} 31.0-0.2 \ldots \ldots$. & $2.30 \mathrm{E}-04$ & \\
\hline OH $32.0-0.5 \ldots \ldots$. & $1.50 \mathrm{E}-03$ & \\
\hline OH $32.8-0.3 \ldots \ldots$. & $1.40 \mathrm{E}-04$ & \\
\hline OH $39.7+1.5 \ldots \ldots \ldots$ & $6.00 \mathrm{E}-05$ & \\
\hline OH $39.9-0.0 \ldots \ldots$. & $6.30 \mathrm{E}-05$ & \\
\hline $\mathrm{OH} 42.3-0.1 \ldots \ldots$. & $1.60 \mathrm{E}-04$ & \\
\hline $\mathrm{OH} 45.5+0.1 \ldots \ldots$. & $2.50 \mathrm{E}-05$ & \\
\hline
\end{tabular}

Notes.-CO mass-loss rates taken from Knapp and Morris (1985) except for OH 26.5+0.6 (van der Veen 1987).

from the choice of our standard dust parameters (see Table 1). Note that, although this will influence the absolute values, this is expected to affect the relative mass-loss rates much less. These uncertainties include the conversion factors from the optical depth to dust column density (i.e., $S[10 \mu \mathrm{m}]$ ) and to gas mass-loss rate (i.e., $\delta$ ). Measured peak strengths of the $10 \mu \mathrm{m}$ feature in silicate materials vary from about $10^{3} \mathrm{~cm}^{2} \mathrm{~g}^{-1}$ for amorphous materials to $10^{4} \mathrm{~cm}^{2} \mathrm{~g}^{-1}$ for more crystalline materials (Day 1979; Penman 1976). Since circumstellar silicates are generally thought to be amorphous, our choice for $S$ $(10 \mu \mathrm{m})$, appropriate for amorphous magnesium-rich silicates, is reasonable and uncertain perhaps by a factor of 2 . The dustto-gas conversion factor assumes that all the available silicon condenses out in the form of silicate grains. Studies of the $\mathrm{SiO}$ emission from oxygen-rich Miras suggest that a large fraction $(99 \%)$ of the available silicon is indeed depleted (Morris et al. 1979). Observations of the strength of the $10 \mu \mathrm{m}$ absorption feature per $\mathrm{H}$ atom in the diffuse interstellar medium also suggest that a large fraction $(\sim 100 \%)$ of the silicon is depleted in the form of silicates (Tielens and Allamandola 1987a). Thus the uncertainty in the silicon depletion factor seems small compared to other uncertainties. Formally, the gas-to-dust conversion factor depends also on the assumed composition of the silicate (e.g., $\mathrm{MgFeSiO}_{4}$ ) through the mass per silicon atom. However, the $10 \mu \mathrm{m}$ strength of the silicate material is correlated to the number of $\mathrm{SiO}_{4}$ tetrahedra present. Thus, adding relatively more $\mathrm{Mg}$ or $\mathrm{Fe}$ will decrease the peak absorption strength per gram of material, and this compensates for the difference in composition. 
Table 2 also gives mass-loss rates for six red giants derived from CO observation (Knapp and Morris 1985). Generally, they are within a factor of 3 of the IR estimates. The large discrepancy between the CO (van der Veen 1987) and IR results for $\mathrm{OH} 26.5+0.6$ will be discussed in $\S \mathrm{Vd}$. We note that the mass-loss rates of IRC 10011 and $\mathrm{OH} 26.5+0.6$ derived from the $A_{10}-T_{c}$ relation are in good agreement with those resulting from a very detailed model fit of the IR spectrum (see $\S \mathrm{V}$ and Table 3).

\section{e) Deviations from the $A_{10}-T_{c}$ Relation \\ i) Interstellar Extinction}

There has been some discussion in the literature that the infrared characteristics of $\mathrm{OH} / \mathrm{IR}$ stars may be affected by interstellar extinction (Evans and Beckwith 1977; Forrest et al. 1978; Werner et al. 1980; Herman 1983; Gehrz et al. 1985). In general, the extreme reddening of $\mathrm{OH} / \mathrm{IR}$ stars is due to circumstellar material as evidenced by the variability of the infrared colors and of the strength of the $10 \mu \mathrm{m}$ silicate absorption feature, by the correlation of colors with period, and by the general absence of interstellar molecular clouds along the line of sight (Evans and Beckwith 1977; Forrest et al. 1978; Werner et al. 1980). In principle, the observed $A_{10}-T_{c}$ relation might, however, be affected to some extent by interstellar extinction, in particular the extreme red OH/IR stars. However, since the well-studied, nearby source $\mathrm{OH} 26.5+0.6$ falls along the trend defined by the others, major effects seem unlikely. The arrows in Figure 4 indicate the effect of adding 30 magnitudes of visual extinction by cold foreground dust, assuming the infrared extinction law observed toward the galactic center (Becklin et al. 1978). The effect of foreground extinction is most pronounced for relatively thin shells. For the most extreme red shells $\left(T_{c} \lesssim 200 \mathrm{~K}\right)$ the extinction correction falls almost parallel to the observed trend. For such cool shells it is obviously difficult to distinguish foreground and interstellar extinction in the $A_{10}-T_{c}$ diagram. For these stars one might do slightly better by using the $20 \mu \mathrm{m}$ absorption feature.

A close examination of Figure 4 suggests that the sources $\mathrm{OH} 0.2+0.0$ and $\mathrm{OH} 4.6-0.4$, which deviate considerably from the trend displayed by the other objects, are probably heavily influenced by interstellar extinction. It is likely that these sources, which are close to the galactic center (i.e., $A_{v} \approx$ $30 \mathrm{mag}$; Becklin et al. 1978), are actually similar to the optically visible Miras in the solar neighborhood. Possibly, AFGL 2885 is also affected by interstellar extinction, although in this case the small displacement from the general trend of the other sources may be due to the use of spectrophotometry rather than filter data to define $A_{10}$. For the other sources, even the heavily obscured $\mathrm{OH} / \mathrm{IR}$ stars, the effects of interstellar extinction seem to be small, contributing on the average perhaps $0.5 \mathrm{mag}$ and $20 \mathrm{~K}$ to the spread in $A_{10}$ and $T_{c}$. This is not surprising, since the average distance of the $\mathrm{OH} / \mathrm{IR}$ stars in our sample is of the order of $5 \mathrm{kpc}$, corresponding to about 10 visual magnitudes of extinction (i.e., $1.9 \mathrm{mag} \mathrm{kpc}-1$; Allen 1973). This results in a $\tau(10 \mu \mathrm{m})$ and $\tau(2 \mu \mathrm{m})$ of about 0.5 and 1.0 , respectively. Because of the steepness of the blackbody function at low temperatures $(\sim 400 \mathrm{~K})$ and short IR wavelengths $(2 \mu \mathrm{m})$, the latter has only a minor effect on the color temperature. We note that, except for the two sources commented upon above, the sample of OH/IR stars close to the galactic center and the tangential point $(D \approx 10 \mathrm{kpc}$; Baud et al. 1985) lies well above the relation of the other objects. Clearly, the differences in photometric color systems used has a greater influence on the spread of the points than the possible presence of interstellar extinction.

\section{ii) Bipolar Reflection Nebulae}

Two more objects, which are displaced from the general $A_{10}-T_{c}$ relation, should be commented on. The first one, $\mathrm{OH}$ $231.8+4.2$ (also known as $\mathrm{OH} 0739-14$ ), has a very deep $10 \mu \mathrm{m}$ absorption feature $\left(A_{10} \approx 1.75\right)$ and a high color temperature $\left(T_{c} \approx 900 \mathrm{~K}\right)$. This M9 III (Cohen 1981) Mira variable (Feast et al. 1983) is embedded in a bipolar infrared reflection nebula which is viewed almost edge-on (Cohen and Frogel 1977; Allen et al. 1980; Tielens, Werner, and Capps 1985) and the comparatively high color temperature results from scattering through the lobes at near-infrared wavelengths. None of the other Miras or OH/IR stars in our sample falls close to it in the $A_{10}-T_{c}$ plot. The second peculiar object is $\mathrm{OH} 17.7-2.0$, which has a very low color temperature $\left(T_{c} \approx 280 \mathrm{~K}\right)$ and yet a very shallow $10 \mu \mathrm{m}$ absorption feature $\left(A_{10} \approx 0.35\right.$; Herman 1983). This object, a K1-K4 giant or supergiant, is probably also embedded in a small bipolar nebula (Le Bertre 1987) and the near-infrared radiation is again due to scattering through the bipolar lobes. While the 1-3 $\mu \mathrm{m}$ color temperature is quite high $(\sim 1700 \mathrm{~K})$ as expected for a heavily obscured scattering nebula powered by a $4000 \mathrm{~K}$ central star, the much lower $3.4-12.5 \mu \mathrm{m}$ color temperature $(280 \mathrm{~K})$ reveals the presence of an extensive dust shell at a relatively large distance of the star $\left(\sim 4 \times 10^{15} \mathrm{~cm}\right)$, which reprocesses most of the stellar radiation. The weak $10 \mu \mathrm{m}$ absorption feature suggests that we are looking almost pole-on into this bipolar nebula. The spectral differences between this source and $\mathrm{OH} 231.8+4.6$ may thus result from a difference in viewing angle. Both sources may be descended from AGB stars and may have recently changed their mass-loss mode from spherically symmetric on the AGB to equatorial symmetry in the protoplanetary nebula phase. Alternatively, it has been suggested that a period of high massloss rate was recently terminated, resulting in a cool detached dust shell (van der Veen, Habing, and Geballe 1987; Bedijn 1987).

\section{DETAILED MODELS OF THE CIRCUMSTELLAR SHELLS OF AGB STARS}

In this section we will discuss detailed model fits to the IR spectra of the AGB stars R Cas, IRC 10011, OH 26.5+0.6. The aim of this section is to determine the intrinsic absorption properties of the dust. In particular, the shape of the $10 \mu \mathrm{m}$ spectral feature is very sensitive to the assumed intrinsic shape of the $10 \mu \mathrm{m}$ silicate feature. Using a trial and error method, the inherent $10 \mu \mathrm{m}$ dust absorption properties can thus be determined from the observed flux distribution. From the derived dust column density in the shell, the stellar mass-loss rate is also determined. Finally, an extensive study has been made on the dependence of the emergent spectrum on variation of the free parameters. The resulting uncertainties in the derived dust absorption properties and the mass-loss rate will be briefly discussed.

\section{a) R Cas}

$\mathrm{R}$ Cas is a long-period Mira variable of spectral type M7e (Forrest, Gillett, and Stein 1975). Its infrared spectrum, taken at phase 0.14 (Fig. 6, Forrest, Gillett, and Stein 1975), shows the $10 \mu \mathrm{m}$ feature in emission, indicative of an optically thin circumstellar dust shell. Its distance, effective temperature, and terminal velocity of the gas are listed in Table 3 , together with 
TABLE 3

Observed and Derived Properties of the Circumstellar ENVELOPES AROUND R CAS, IRC 10011, AND OH 26.5 + 0.6

\begin{tabular}{|c|c|c|c|c|}
\hline Parameter & $\mathrm{R}$ Cas & IRC 10011 & OH $26.5+0.6$ & $\begin{array}{l}\text { References } \\
\text { and Notes }\end{array}$ \\
\hline$D(\mathrm{kpc}) \ldots \ldots \ldots \ldots$ & 0.28 & 0.47 & 0.98 & 1,2 \\
\hline$T_{\text {eff }}(\mathrm{K}) \quad \ldots \ldots \ldots \ldots$ & 2500 & 1800 & 2000 & \\
\hline$v_{\infty}\left(\mathrm{km} \mathrm{s}^{-1}\right)$ & $\left\{\begin{array}{r}8.3 \\
12.3\end{array}\right.$ & 10.0 & 14.1 & $\begin{array}{l}\text { 5, } 6 ; \mathrm{OH}^{\mathrm{a}} \\
7 ; \mathrm{CO}^{\mathrm{a}}\end{array}$ \\
\hline$P$ (days) .......... & 431 & & 1630 & 3,8 \\
\hline$y_{0} \ldots \ldots \ldots \ldots \ldots$ & 0.89 & 0.99 & 0.98 & \\
\hline$T_{s}(\mathrm{~K}) \ldots \ldots \ldots \ldots$ & 1000 & 1000 & 1000 & \\
\hline$Q_{0} / Q_{\text {peak }} \cdots \ldots \ldots \ldots$ & 0.49 & 0.49 & 0.40 & \\
\hline$\tau(10 \mu \mathrm{m}) \ldots \ldots \ldots$ & 0.10 & 4.60 & 28.0 & \\
\hline$N_{d}\left(\mathrm{~cm}^{-2}\right) \ldots \ldots \ldots$ & $3.8(10)$ & $1.8(12)$ & $1.1(13)$ & b \\
\hline$r_{0} / r_{*} \ldots \ldots \ldots \ldots \ldots$ & 4.6 & 3.0 & 5.8 & \\
\hline$r_{*}\left(10^{13} \mathrm{~cm}\right) \ldots \ldots$ & 6.3 & 5.2 & 6.9 & \\
\hline$L_{* *}^{*}\left(10^{4} L_{\odot}\right) \quad \ldots \ldots$ & 2.9 & 0.52 & 1.4 & \\
\hline$\dot{M}\left(M_{\odot} \mathrm{yr}^{-1}\right) \ldots$ & $2.9(-7)$ & $1.9(-5)$ & $2.2(-4)$ & \\
\hline Dust model $\ldots \ldots$ & 3 & 2 & 1 & \\
\hline
\end{tabular}

REFERENCES.-11) Nguyen-Q.-Rieu et al. 1979; (2) Herman 1983; (3) Forrest et al. 1975; (4) Hyland et al. 1972; (5) Olnon 1977; (6) Jewell et al. 1980; (7) Knapp and Morris 1985; (8) Engels 1982.

${ }^{a} \mathrm{OH}$ and $\mathrm{CO}$ refer to $v_{\infty}$ data obtained from observations of these molecules.

${ }^{\mathrm{b}}$ Calculated assuming a grain size of $500 \AA$.

the values that were assumed for the sublimation temperature, $y_{0}$ and $Q_{0} / Q_{\text {peak }}$ in our standard model for this object. The stellar radius and luminosity have been calculated from the measured $3.5 \mu \mathrm{m}$ magnitude (Forrest, Gillett, and Stein 1975). The terminal gas velocity was taken equal to the $\mathrm{OH}$-maser velocity. To evaluate $y_{0}$, the parameter determining the steepness of the density distribution near the inner edge of the dust shell (see eq. [5] and [17]), we adopted the $\mathrm{OH}$ outflow veloc-

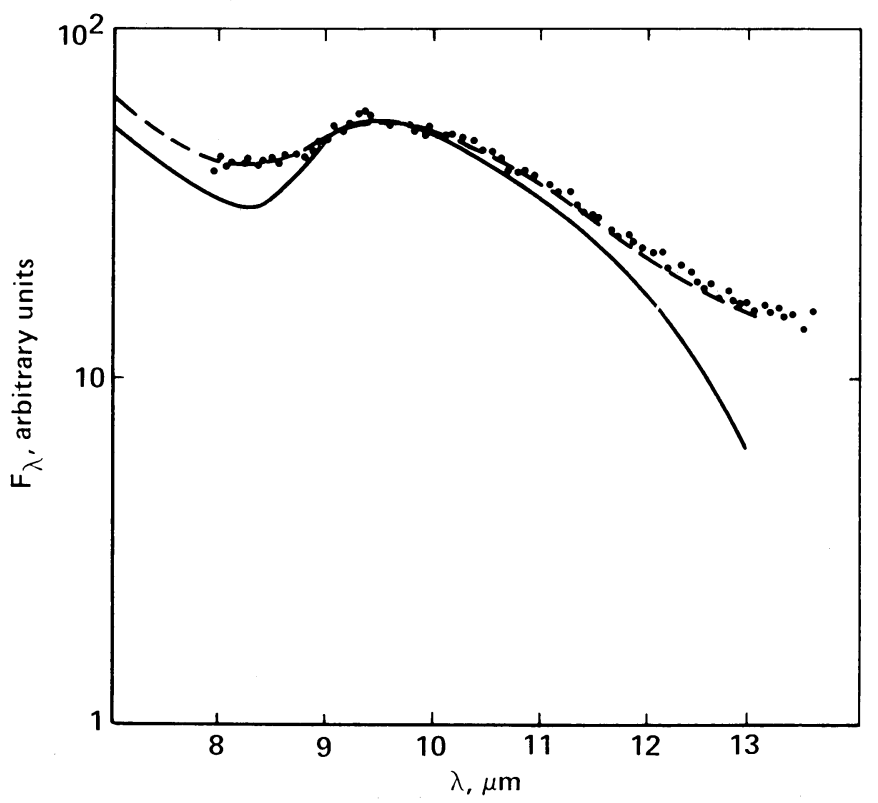

Fig. 6 ity and assumed an initial gas velocity at the dust sublimation radius $r_{0}$ of $2.8 \mathrm{~km} \mathrm{~s}^{-1}$, which is about equal to the sound velocity in an $\mathrm{H}_{2}$ gas at the typical gas temperature in the dust condensation zone (800 K; Hinkle, Hall, and Ridgway 1982).

With these parameters a good fit of the observed $8-13 \mu \mathrm{m}$ spectrum was found with dust model 3 (see Fig. 2) for $\tau(10 \mu \mathrm{m})=0.10$ (Fig. 6). The absorption properties of this model around $10 \mu \mathrm{m}$ are very similar to the $10 \mu \mathrm{m}$ feature shape found from observations of Orion (Gillett et al. 1975), except for a slightly steeper short wavelength wing. It should be emphasized that dust models 1 and 2 (Fig. 2), which fit the observed spectra of $\mathrm{OH} 26.5+0.6$ and IRC 10011, respectively, do not provide good fits to the observed spectrum of $\mathbf{R}$ Cas. This is illustrated for dust model 1 in Figure 6 . In view of the discussion in $\S \mathrm{II} b$ and Appendix A, the derived intrinsic shape of the $10 \mu \mathrm{m}$ silicate feature is expected to be insensitive to variation in the other free parameters. This is borne out by detailed numerical calculations. For example, a fit of the $10 \mu \mathrm{m}$ feature almost identical to the fit shown in Figure 6 is obtained with $T_{s}=750 \mathrm{~K}$ and $\tau(10 \mu \mathrm{m})=0.073($ see $\S$ III $b)$. A good fit to the observed spectrum can also be obtained for different $Q_{0} / Q_{\text {peak }}$ values by adjusting the value of $\tau(10 \mu \mathrm{m})$ appropriately.

Conversely, the spectrum of $\mathrm{R}$ Cas around $10 \mu \mathrm{m}$ alone does not constrain the values of $T_{s}, y_{0}$, and $Q_{0} / Q_{\text {peak }}$ very much. In fact, within a wide range of adopted values, a good fit can be obtained when the $10 \mu \mathrm{m}$ shell opacity is allowed to be chosen freely. It should be emphasized that the differences in the shape of the infrared spectrum shortward of about $6 \mu \mathrm{m}$ resulting from varying $T_{s}$ and $Q_{0} / Q_{\text {peak }}$ are small. In this region the emergent spectrum is completely dominated by the stellar flux which is only slightly attenuated by the optically thin shell. For example, in our standard model the $2 \mu \mathrm{m}$ optical depth is only 0.05. Our models show that allowing reasonable values for the dust sublimation temperature $\left(T_{s}=1000 \pm 300 \mathrm{~K}\right)$, and an

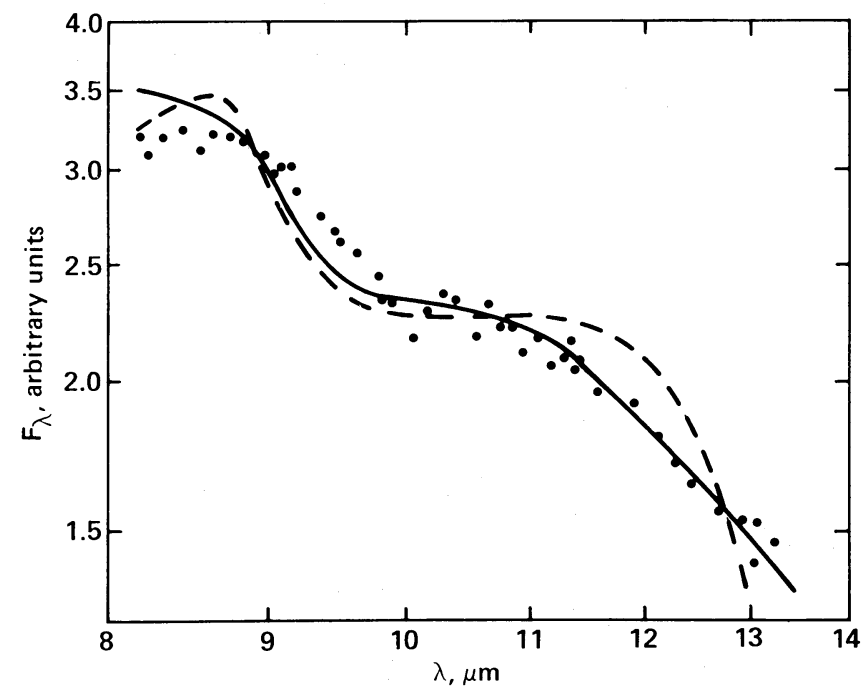

FIG. 7

Fig. 6.-A comparison of the model calculations with the 8-13 $\mu$ m observations toward R Cas (Forrest $e t$ al. 1974). The dashed line gives the best fit using dust model 3 from Fig. 2. The solid line gives the best fit possible with dust model 1 from Fig. 2.

FIG. 7.-A comparison of the 8-13 $\mu \mathrm{m}$ spectrometric observations of IRC 10011 (Merrill and Stein 1976b) with the model calculations. The solid line gives the best-fit model using dust model 2 from Fig. 2 . The dashed line is the best possible fit with dust model 1. 
uncertainty in $Q_{0} / Q_{\text {peak }}$ of about $30 \%$, results in an uncertainty in $\tau(10 \mu \mathrm{m})$ of about $40 \%$.

The mass-loss rate (see Table 3 ) has been derived using the values of $\tau(10 \mu \mathrm{m})$ and $y_{0}$ of the standard model. The model with $T_{s}=750 \mathrm{~K}$ has a $70 \%$ greater condensation distance $r_{0}$, but this is partly compensated by its smaller $\tau(10 \mu \mathrm{m})$ and the mass-loss rate is only $25 \%$ higher. Similarly, $Q_{0} / Q_{\text {peak }}=0.25$ requires a $50 \%$ larger $10 \mu \mathrm{m}$ optical depth and a $13 \%$ smaller condensation radius. This leads to a $40 \%$ larger mass-loss rate. Equation (20) also shows a dependence of $\dot{M}$ on $y_{0}$; that is, on the steepness of the density distribution near the inner boundary. For $y_{0}$ is 0.64 , which is much smaller than reasonably can be expected (i.e., $v_{0}=5.0 \mathrm{~km} \mathrm{~s}^{-1}$ ), the mass-loss rate is only $20 \%$ higher. Similarly, for $y_{0}=0.97$, corresponding to the adopted $v_{0}$ of $2.8 \mathrm{~km} \mathrm{~s}^{-1}$ and the CO outflow velocity, $\dot{M}$ is only $13 \%$ lower. In conclusion, the derived value of the massloss rate, $3 \times 10^{-7} M_{\odot} \mathrm{yr}^{-1}$, is uncertain by about $50 \%$.

\section{b) IRC 10011}

IRC 10011 is a late $M$ giant (Hyland et al. 1972; Dyck, Lockwood, and Capps 1974) which is known to be a Mira variable. Its spectrum shows a $10 \mu \mathrm{m}$ emission feature which displays self-absorption in its center (Merrill and Stein 1976b; Fig. 7), indicative of a fairly thick circumstellar shell. The assumed values of its effective temperature, distance, and gas velocity at infinity, taken equal to the observed $\mathrm{CO}$ outflow velocity, are listed in Table 3. This table contains also our assumptions for the sublimation temperature, $y_{0}$ and $Q_{0} / Q_{\text {peak }}$. With these parameters and using dust model 2 we find a bestfit model spectrum for $\tau(10 \mu \mathrm{m})=4.6$ which in Figure 8 is compared with the observed overall spectral distribution given by photometric observations. Although these data correspond to different phases and luminosities of the stellar source, they do not seem to indicate a significant variation in the spectral distribution of IRC 10011. Therefore, we have scaled all the data to the same luminosity. The model spectrum fits the data very well.

The analysis of the intrinsic shape of the $10 \mu \mathrm{m}$ dust feature is complicated somewhat by the presence of self-absorption in the $10 \mu \mathrm{m}$ spectrum of this source (Fig. 7). The intrinsic shape of dust model 2 (see Fig. 2) gives a good, although not perfect, fit of the spectrophotometric data. Both the sharper feature of dust model 1 as well as the broader feature of dust model 3 (see Fig. 2) give, when normalized at $10 \mu \mathrm{m}$, a higher flux around $12 \mu \mathrm{m}$ than dust model 2. This is illustrated in Figure 7 for dust model 1. Although the emergent spectrum around $10 \mu \mathrm{m}$ will also depend somewhat on the values of $T_{s}$ and $Q_{0} / Q_{\text {peak }}$, a better fit to the data around $12 \mu \mathrm{m}$ is not possible for dust models 1 and 3, and dust model 2 is preferred for this source.

In contrast to R Cas, the near-infrared ( $\lesssim 5 \mu \mathrm{m}$ ) spectrum of IRC 10011 is sensitive to the choice of $Q_{0} / Q_{\text {peak }}$ and $T_{s}$. For example, decreasing $T_{s}$ or increasing $Q_{0} / Q_{\text {peak }}$ results in a decrease of the near-infrared flux (see Appendix A, Fig. 15). Although variations in $T_{s}$ and $Q_{0} / Q_{\text {peak }}$ can have opposing effects, they do not completely cancel. In particular, there is a contribution around $3 \mu \mathrm{m}$ from hot dust in our standard model, and its absence in lower $T_{s}$ models cannot easily be corrected for by varying the near-infrared opacity, unless we depart from the simple absorption law adopted (see eq. [6]). This is, however, beyond the scope of this article.

Observations of the angular distribution of the intensity are a potentially powerful tool for determining the structure of circumstellar dust shells. At short wavelengths $(\lambda<3 \mu \mathrm{m})$ the intensity distribution will reflect the morphology of the hot (or scattering) grains near the inner boundary, while at longer wavelengths the cooler outer portions of the shell will dominate. The angular intensity distribution of IRC 10011 has been measured at $2.2,10$, and $20 \mu \mathrm{m}$ using lunar occultation techniques (Zappala et al. 1974; see Fig. 9). As expected, emission at large wavelengths originates at greater distances from the stellar source from dust at lower temperature. Model curves have been calculated of the intensity distribution from the Fresnel diffraction pattern along a straight edge. For the broad-band filters at 10 and $20 \mu \mathrm{m}(8-13$ and $17-24 \mu \mathrm{m})$ model occultation curves at sampled wavelengths were calculated and summed using the appropriate weighting function.

It is expected that $T_{s}$ will influence the intensity distribution at short wavelengths considerably. Theoretical intensity distribution functions have been calculated for two models with different $T_{s}(1000 \mathrm{~K}$ vs. $750 \mathrm{~K}$; see Fig. 9). The assumed stellar luminosity and thus $r_{*}$ (and $r_{0}$ ) has been slightly reduced (i.e. $4.6 \times 10^{3} L_{\odot}$ and $\left.4.9 \times 10^{13} \mathrm{~cm}\right)$ to agree with photometric

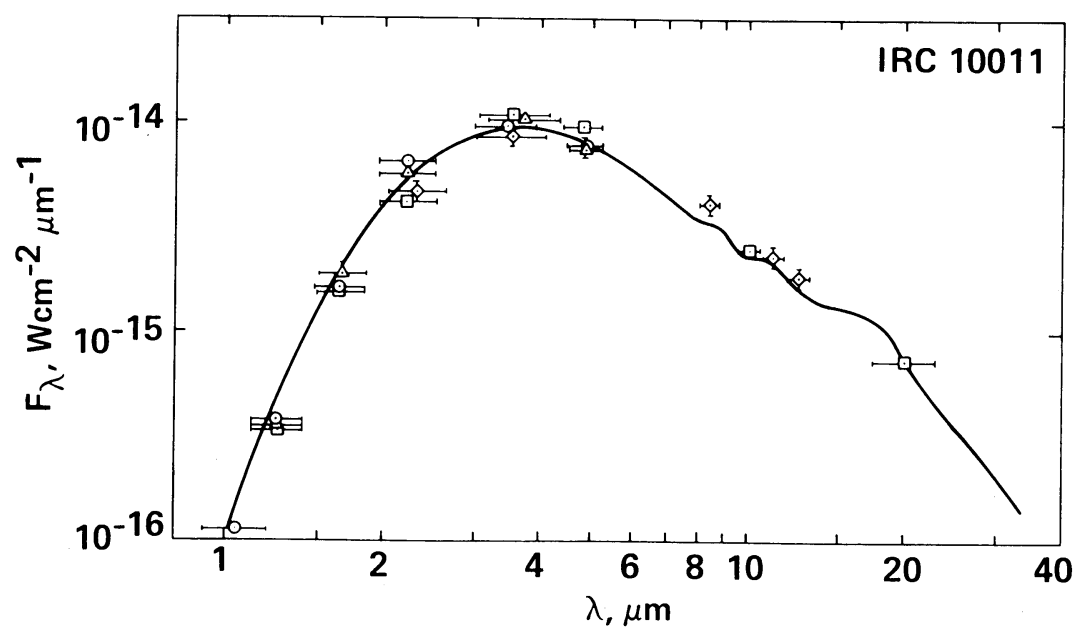

FIG. 8.-A comparison of the photometric observations of IRC 10011 with the model calculations. Data were taken from Dyck et al. $(1974)(\bigcirc)$, Engels $1982(\triangle)$, Zappala et al. $1974(\square)$, and Merrill and Stein $1976 b(\diamond)$. The flux levels of these observations were multiplied by $1.97,1.51,0.88$, and 1.0 , respectively, to scale them to the same absolute luminosity (see text). The solid line gives the best-fit model obtained with dust model 2 from Fig. 2. 


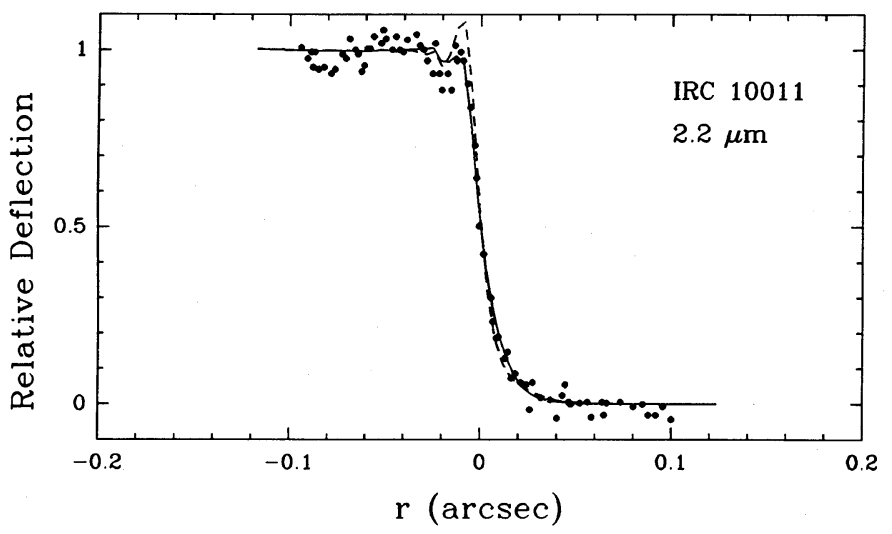

FIG. $9 a$

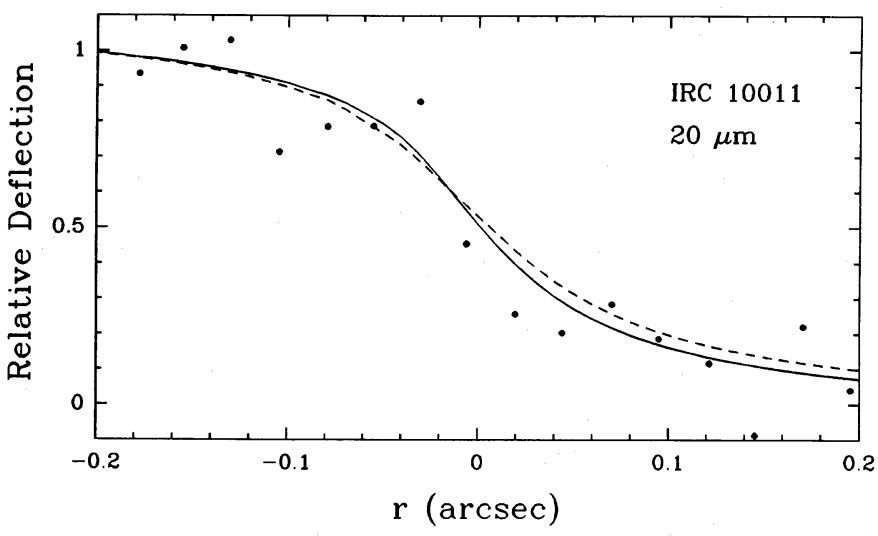

Fig. $9 c$

Fig. 9.-Normalized disappearance curves of IRC 10011 as a function of angular separation from the lunar limb at $2.2 \mu \mathrm{m}(a), 10 \mu \mathrm{m}(b)$, and $20 \mu \mathrm{m}(c$; Zappala et al. 1974) compared with simulated curves for our model intensity distributions. The solid line corresponds with the standard model having $T_{s}=$ $1000 \mathrm{~K}$, the dashed line corresponds with the $T_{s}=750 \mathrm{~K}$ model.

data obtained at the same time. Note that the spectral shape is insensitive to the phase of the star (see Fig. 8). The other free parameters have been varied to obtain the best possible fit to the observed spectrum for each case. Although at $2.2 \mu \mathrm{m}$ the $T_{s}=1000 \mathrm{~K}$ fits the data better, a lower inner dust shell temperature cannot be excluded, the more since scattering may be important at this wavelength. However, the $10 \mu \mathrm{m}$ data clearly favor the $T_{s}=1000 \mathrm{~K}$ model. The larger scatter in the $20 \mu \mathrm{m}$ observations preclude any meaningful comparison with the model calculations.

We estimate the overall uncertainty in the derived values of $T_{s}$ and $Q_{0} / Q_{\text {peak }}$ to be about $10 \%$. The overall uncertainty in $\tau(10 \mu \mathrm{m})$ is then estimated to be about $15 \%$ and stems mainly from $T_{s}$ and $y_{0}$, as variations in $Q_{0} / Q_{\text {peak }}$ have little influence on the spectrum around $10 \mu \mathrm{m}$. Using equation (20) a massloss rate of $1.9 \times 10^{-5} M_{\odot} \mathrm{yr}^{-1}$ is derived. Since the uncertainties in $\tau(10 \mu \mathrm{m})$ are partly compensated by resulting variations in $r_{0}$ or $y_{0}$, the uncertainty in $\dot{M}$ is fairly small $(\sim 10 \%)$. The main uncertainty in $\dot{M}$ results from the conversion of optical depth to dust column density $(S[10 \mu \mathrm{m}])$ and the dust-to-gas mass-loss rate (i.e., $\delta$ ).

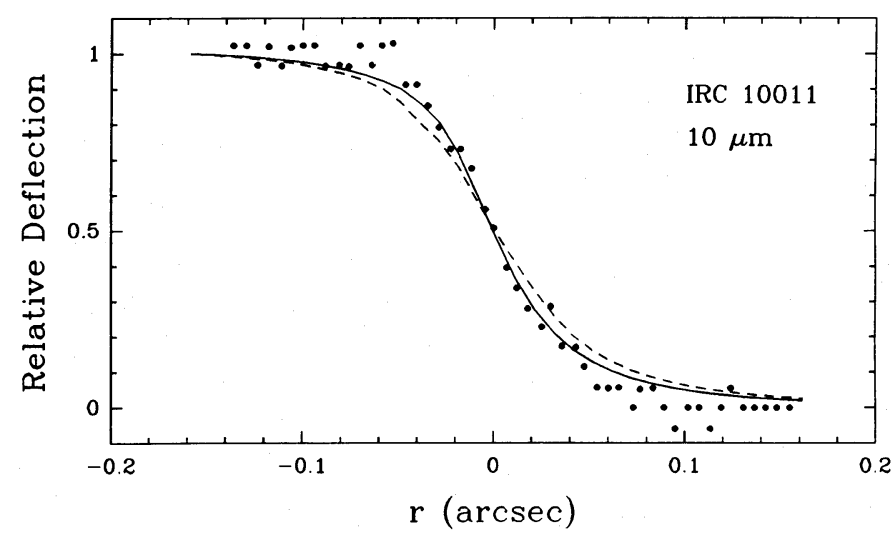

FIG. $9 b$

c) $O H / I R 26.5+0.6$

$\mathrm{OH} / \mathrm{IR} 26.5+0.6$ is a strong infrared source (AFGL 2205) which shows long-period variability and is associated with a bright $1612 \mathrm{MHz}$ OH maser. Its spectrum shows a deep $10 \mu \mathrm{m}$ absorption feature, indicating a highly optically thick dust shell. The standard values that were adopted for its distance, effective temperature, outflow velocity, $y_{0}$, sublimation temperature, and $Q_{0} / Q_{\text {peak }}$ are listed in Table 3, together with the period as deduced from IR observations. A direct observational determination of the effective temperature of the central stellar source is impossible due to the high opacity of the shell. Therefore we have adopted $T_{\text {eff }}=2000 \mathrm{~K}$, a typical value for AGB stars. The uncertainty in this value is, however, unimportant for the emergent spectrum (see Appendix A). Figures 10 and 11 show the best-fit model found for $\tau(10 \mu \mathrm{m})=28.0$ with dust model 1. A comparison is made with photometric data obtained by several investigators (Evans and Beckwith 1977; Werner et al. 1980; Engels 1982) at nearly equal total luminosity (Fig. 10). The model fits the data generally very well, but there are some deviations around 3.6 and longward of $14 \mu \mathrm{m}$. The implications of this will be discussed after we have assessed the influence of the free parameters on the spectral distribution.

Dust model 1 gives a satisfying fit of the $10 \mu \mathrm{m}$ spectroscopic observations (Fig. 11) which were obtained at nearly the same total luminosity as the photometric data displayed in Figure 10. The best possible fit with an intrinsic shape of the $10 \mu \mathrm{m}$ dust feature derived from the observed $10 \mu \mathrm{m}$ spectrum of the Trapezium stars in Orion (Gillett et al. 1975) is also shown. This dust model is very similar to dust model 3 (Fig. 2). Clearly, the Orion dust model gives a far too broad $10 \mu \mathrm{m}$ absorption, due to shallowness of its $10 \mu \mathrm{m}$ resonance. Similarly, dust model 2 and 3 fail to fit the shoulders of the observed $10 \mu \mathrm{m}$ feature.

As was the case for IRC 10011, the near-infrared $(\lambda<5 \mu \mathrm{m})$ spectrum depends strongly on the choice for $T_{s}$ and $Q_{0} / Q_{\text {peak }}$. From extensive parameter studies, we conclude that the standard values for these two parameters (see Table 3) are uncertain by about $10 \%$. The variations in the value of $Q_{0} / Q_{\text {peak }}$ between $\mathrm{OH} 26.5+0.6$ and IRC 10011 seem thus to be real. The resulting uncertainty in $\tau(10 \mu \mathrm{m})$ amounts then to about $30 \%$. The stellar effective temperature has no influence on the emergent spectrum, due to the high obscuration by the envelope. Note that $T_{\text {eff }}$ has also no influence on $r_{0}$ since the heating at the inner boundary is dominated by backheating from the envelope (see Appendix A $e$ ). The mass-loss rate derived from our models is about $2 \times 10^{-4} M_{\odot} \mathrm{yr}^{-1}$. Again 


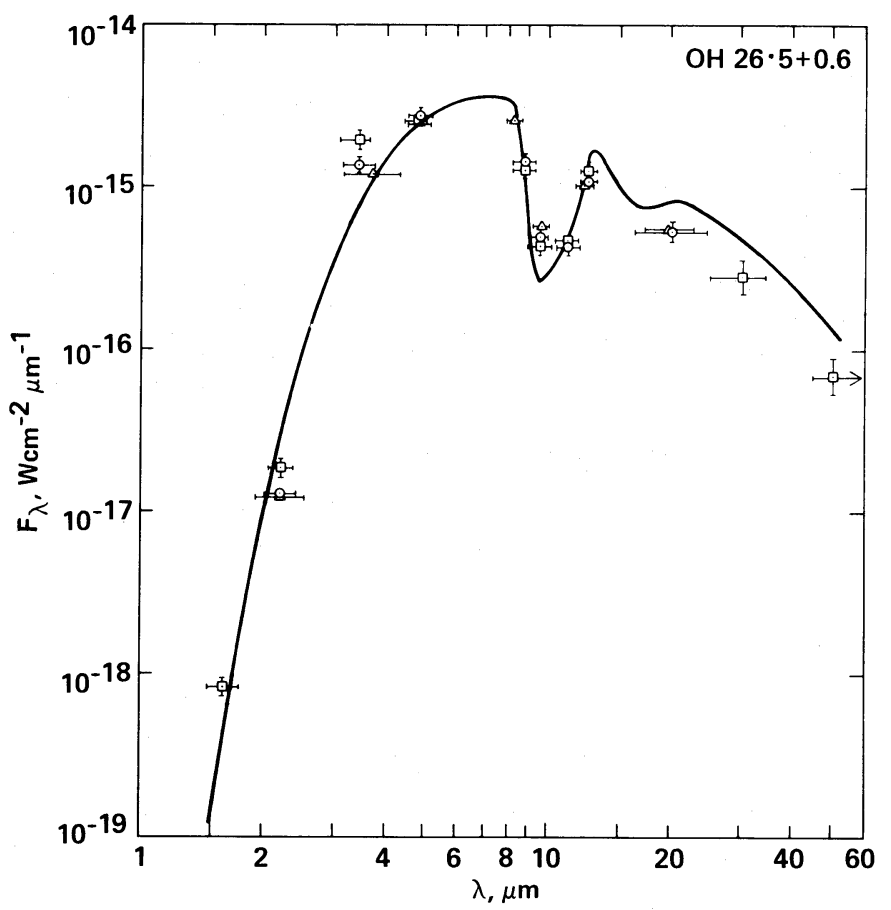

FIG. 10.-A comparison of the photometric observations of $\mathrm{OH} / \mathrm{IR}$ $26.5+0.6$ with the model calculations. Data were taken from Engels $1982(\triangle)$; Werner et al. $1980(\square)$, and Evans and Beckwith $1977(\bigcirc)$. The solid line gives the best-fit model found with dust model 3 of Fig. 2 .

variations in $T_{s}$, which are the dominant source of uncertainty for the mass-loss rate in the radiative transfer model, are partially offset by variations in $r_{0}$ required to fit the observed spectrum, resulting in a $15 \%$ uncertainty. The mass-loss rate depends also on the assumed density distribution at the inner boundary (e.g., $y_{0}$ ). However, this is largely compensated by accompanying variations in $\tau(10 \mu \mathrm{m})$, and the uncertainty in $\dot{M}$ due to all free parameters is estimated to be about $20 \%$. This does not include the uncertainty due to the assumed dustto-gas mass-loss conversion factors (a factor of about 3; see $\S \mathrm{IV} d)$.

Although the overall agreement between the observations and our standard model is quite impressive, there are small differences between the observed flux and our standard model around $3.4 \mu \mathrm{m}$ and beyond $20 \mu \mathrm{m}$, pointing toward some difference in dust properties. The near-infrared difference might indicate less absorption around $3.4 \mu \mathrm{m}$ (e.g., a flatter absorption law between $3.4 \mu \mathrm{m}$ and the $10 \mu \mathrm{m}$ silicate feature) and, thus, a steeper absorption law between 3.4 and $2.2 \mu \mathrm{m}$. Recall that model fits of the spectrum of IRC 10011 gave a similar indication (see $\S \mathrm{Vb}$ ). This is not unexpected if the enhanced near-infrared absorption is due to broad electronic absorption bands of transition metals dissolved in silicates (see $\S$ III $c$ ). This near-infrared steepening of the extinction law may also indicate that scattering is more important than assumed. Likewise, the mid-IR observations suggest a somewhat lower absorption (e.g., emission) efficiency beyond $20 \mu \mathrm{m}$ than assumed in our models $\left(Q_{2} / Q_{\text {peak }} \approx 0.26\right.$; see eq. [9]). Recent studies of $I R A S$ far-IR observations of OH/IR stars support this conclusion (Bedijn 1987; Volk and Kwok 1988). However, it can be seen that the shape of the calculated emergent flux distribution is in good agreement with the observations, lending some support for the assumed $\lambda^{-1}$ absorption law. A more extensive study of a larger sample of objects is required to confirm it.
Forrest et al. (1978) actually obtained spectrophotometric data at two different phases, which show that the depth of the $10 \mu \mathrm{m}$ silicate band increases by $60 \%$ when the luminosity decreases by $50 \%$. The effect of stellar variability has been studied by lowering the stellar luminosity while keeping the stellar radius constant (i.e., $T_{\text {eff }} \approx 1700 \mathrm{~K}$ ) as expected for Mira variables (Wallerstein 1977). The effective temperature has, however, no influence on the emergent spectrum, since the shell is highly optically thick (see Appendix Ae). The density structure of the envelope has been assumed to be independent of the stellar phase. The temperature structure of the envelope varies, however, due to the decrease in luminosity. In particular, the dust temperature at the inner radius is $830 \mathrm{~K}$ in this calculation. This global decrease in the dust temperature causes an increased strength of the $10 \mu \mathrm{m}$ absorption feature. The results of this model compare very well to the spectrophotometric data in Figure 12. Other choices are, however, possible. For example, assuming a constant dust condensation temperature (i.e., a phase-dependent inner radius) yields an almost identical spectrum. Although the density structure in the outer shell will not change (i.e., $n_{d} \sim \dot{M} r^{-2}$ for $r \gg r_{0}$; see eqs. [17] and [18], the optical depth through the envelope will increase with decreasing stellar luminosity $\left(N_{d} \sim \dot{M} / r_{0} ;\right.$ see eq. [19]), resulting in an overall cooler spectrum. In any case, the good agreement in the 8-13 $\mu$ m region (see Fig. 12) lends support to our (dust) model for this source.

Angular diameter measurements of $\mathrm{OH} 26.5+0.6$ have been made by Dyck et al. (1984) and Fix and Cobb (1988, Fig. 13). The model curves (see Fig. 13) have been determined by averaging model fluxes at sampled wavelengths and by calculating the visibility curves using the Hankel transformation function (Rogers and Martin 1984). They are normalized to provide an optical match to the observations. At $3.8 \mu \mathrm{m}$ there is a good agreement between the observed model visibility curves. Although there is good agreement between the model

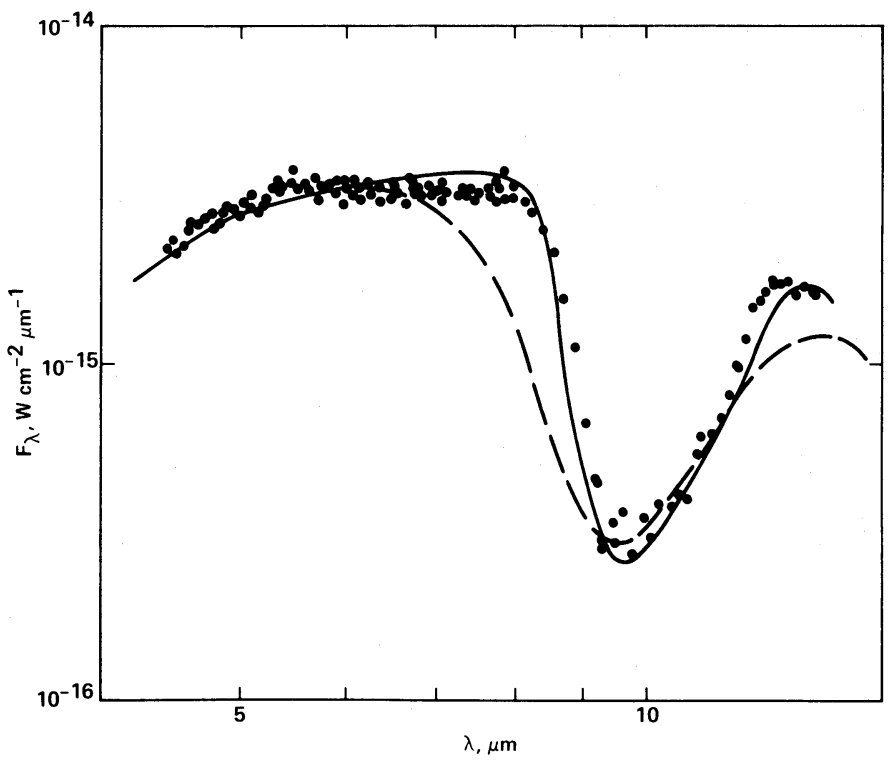

FIG. 11.-A comparison of the model calculations with the spectrometric data of Forrest et al. (1978) of OH/IR 26.5+0.6. The solid line gives the best model fit, assuming dust model 1 from Fig. 2. The dashed line gives the best obtainable model fit using the Orion $10 \mu \mathrm{m}$ feature (Gillett et al. 1975). 


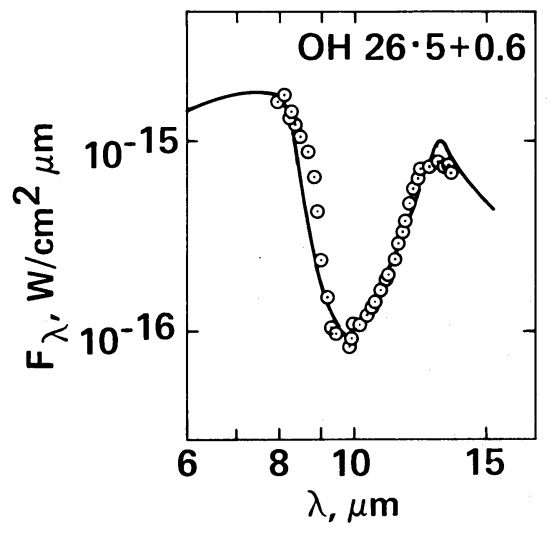

FIG. 12.-A comparison of the model result with the spectrometric data of $\mathrm{OH} / \mathrm{IR} 26.5+0.6$ (Forrest et al. 1978). These data were taken at a luminosity of about half that of the data of Fig. 11. The solid curve gives the model spectrum obtained by assuming the same density distribution as for the standard model, but a lower luminosity (see text).

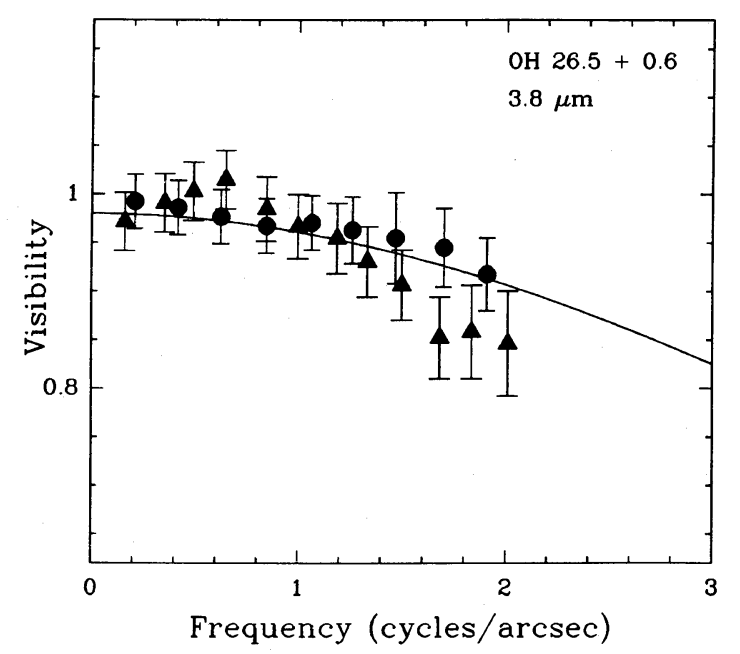

FIG. $13 a$

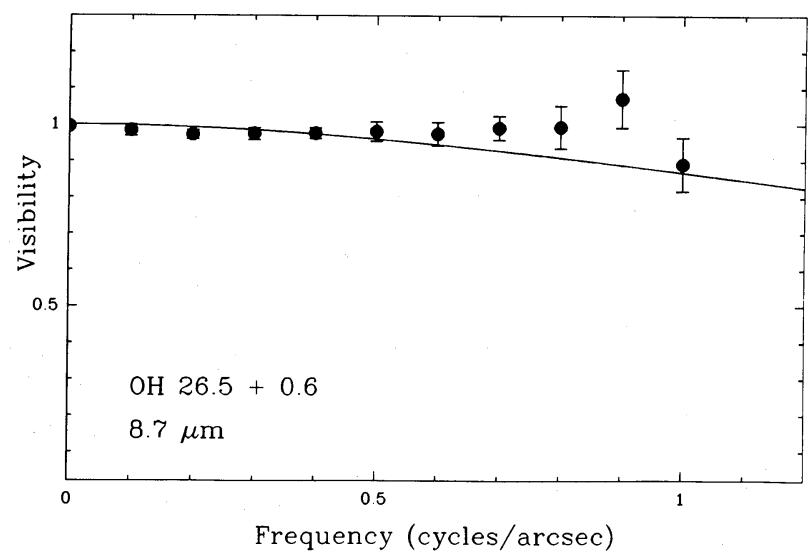

FIG. $13 c$ and the E-W observations at $4.8 \mu \mathrm{m}$, the N-S data however clearly deviate. The data indicate considerable elongation (NS/ $\mathrm{EW}=1.5 \pm 0.25$ ). The sharp increase in size with wavelength between 3.8 and $4.8 \mu \mathrm{m}$ in the N-S direction is somewhat puzzling, since these wavelengths are so close that the same dust should be responsible for the emission. Indeed the calculated sizes at 3.8 and $4.8 \mu \mathrm{m}$ are nearly equal. Clearly, further observational studies are warranted here. Both the 8.7 and $9.8 \mu \mathrm{m}$ data are fitted rather well by the model. The small increase in the visibility at about 0.9 cycles $\operatorname{arcsec}^{-1}$ may indicate a departure from the assumed smooth density distribution at the shortest observed scale length $\left(\sim 0.2^{\prime \prime}\right)$.

Finally, the analytical density distribution derived in $\S$ III (eq. [17]) is strictly speaking correct only for optically thin envelopes where all grains are driven by the same (stellar) radiation field. For optically thick envelopes, such as that around $\mathrm{OH} / \mathrm{IR} 26.5+0.6$, the local radiation field differs considerably from the stellar radiation field and this assumption breaks down. Using our radiative transfer model, we have calculated the second-order density distribution by evaluating the mean of the radiation pressure efficiency, $Q_{r p}$, using the local flux distribution rather than the stellar flux distribution. The velocity can then be calculated by integrating the momentum equa-

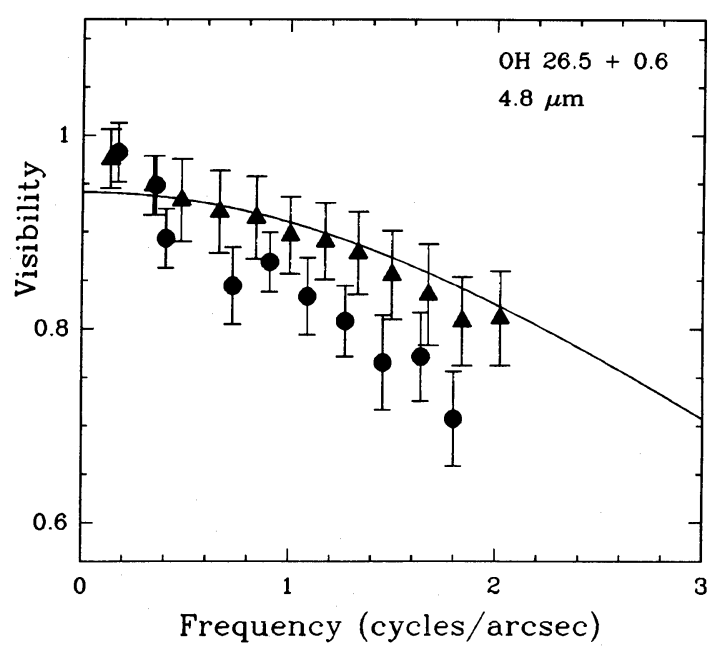

Fig. $13 b$

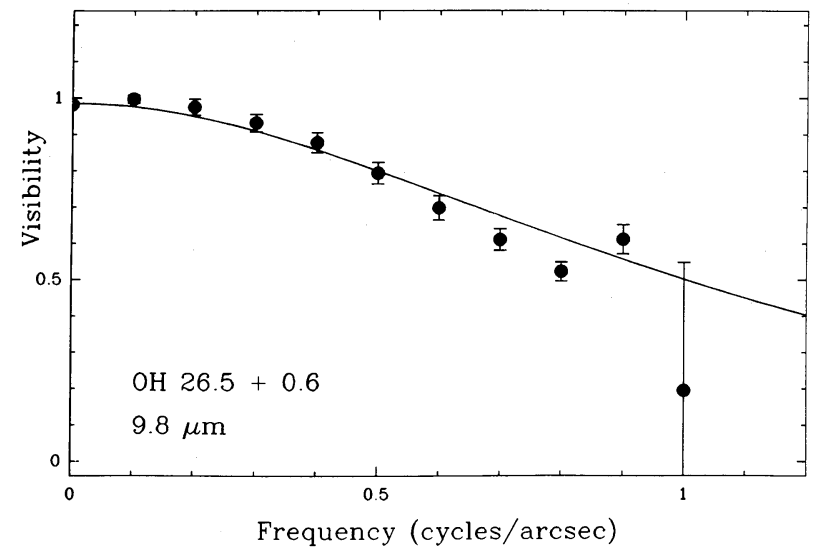

FIG. $13 d$

FIG. 13. - A comparison between the visibility curves of OH/IR $26.5+0.6$ at $3.8 \mu \mathrm{m}(a), 4.8 \mu \mathrm{m}(b), 8.7 \mu \mathrm{m}(c)$, and $9.8 \mu \mathrm{m}(d)$; Dyck et al. 1984; Fix and Cobb $1988)$ and our model curves. Triangles and circles indicate E-W and N-S crosscuts, respectively. For details see text. 
tion numerically (see eqs. [12] and [13]). The resulting second-order density distribution is compared to the analytical results in Figure 14. The second-order approximation is somewhat sharper peaked near the inner boundary. However, the differences are only minor. Essentially, $Q_{r p}$ does not vary much in the envelope due to the dominance of the $10 \mu \mathrm{m}$ feature in the extinction curve and the enhanced near-infrared absorption efficiency. In fact, between 200 and $1000 \mathrm{~K}$ the Planckian average of the absorption efficiency shows a gray behavior. Because the differences between the first- and second-order approximation are so small, higher order correction factors are also expected to be small. This gray behavior implies also that frequency diffusion is not as important as sometimes assumed when deriving mass-loss rates from the momentum equation $(\operatorname{see} \S \mathrm{V} d)$.

\section{d) Discussion}

Calculations on the radiative transfer in the dust shells of three asymptotic giant branch stars have yielded dust properties which are rather uniform, but with some significant differences. Dust properties consistent with the observations are generally characterized by an absorption efficiency with a resonance peak at about $9.7 \mu \mathrm{m}$ and increasing with frequency shortward of $6 \mu \mathrm{m}$ (approximately $\lambda^{-1}$ ). Furthermore, both for IRC 10011 and for OH/IR 26.5+0.6 the model yielded a dust condensation temperature of about $1000 \mathrm{~K}$. For R Cas this parameter is not very well constrained. There are also some differences in the derived dust properties. Most importantly, there seems to be a sequence in the intrinsic shape of the $10 \mu \mathrm{m}$ resonance, going from a broad feature to increasingly sharp features when going from the thin shell of $\mathrm{R}$ Cas to the increasingly thicker shells of IRC 10011 and OH/IR 26.5+0.6 (compare Figs. 6, 7, and 11). Variations in the intrinsic shape of the $10 \mu \mathrm{m}$ silicate feature have also been established for $\mu$ Cep (Rogers, Martin, and Crabtree 1983) and several late-type giants with optically thin dust shells (Pégourie and Papoular 1985).

The derived variations in the intrinsic shape of the $10 \mu \mathrm{m}$ silicate absorption feature between the studied objects are not due to differences in the size of the condensing particles as suggested by Pégourie and Papoular (1985). First, the differences at $13 \mu \mathrm{m}$ are greater than those at $8 \mu \mathrm{m}$, opposite to what is expected for size-related variations. Second, the derived ratio of the near-infrared to the $10 \mu \mathrm{m}$ efficiency varies only little between the three sources, again indicating that a change in size is not the dominant factor. Instead these differences in the intrinsic shape of the $10 \mu \mathrm{m}$ absorption feature are probably related to variations in the optical constants of the silicate material. Such variations could be caused by variations in the temperature or condensation history of the grains. In the calculations it has been assumed that the dust absorption properties are temperature-independent. Actually, however, vibrational absorption features sharpen with decreasing temperature (Plendl 1970; Tielens and Allamandola 1987a). Since the average temperature of the dust observed at $10 \mu \mathrm{m}$ decreases with increasing optical depth of the circumstellar shell, temperature-dependent optical constants of the dust might be able to explain the observations. Unfortunately, no quantitative data on silicates are presently available to test this hypothesis further. We note that this hypothesis has also been put forward (Tielens and Allamandola 1987a) to explain the larger intrinsic width of the $10 \mu \mathrm{m}$ silicate feature observed in emission in Orion $\left(T_{\mathrm{em}} \approx 250 \mathrm{~K}\right)$; as for optically thin dust

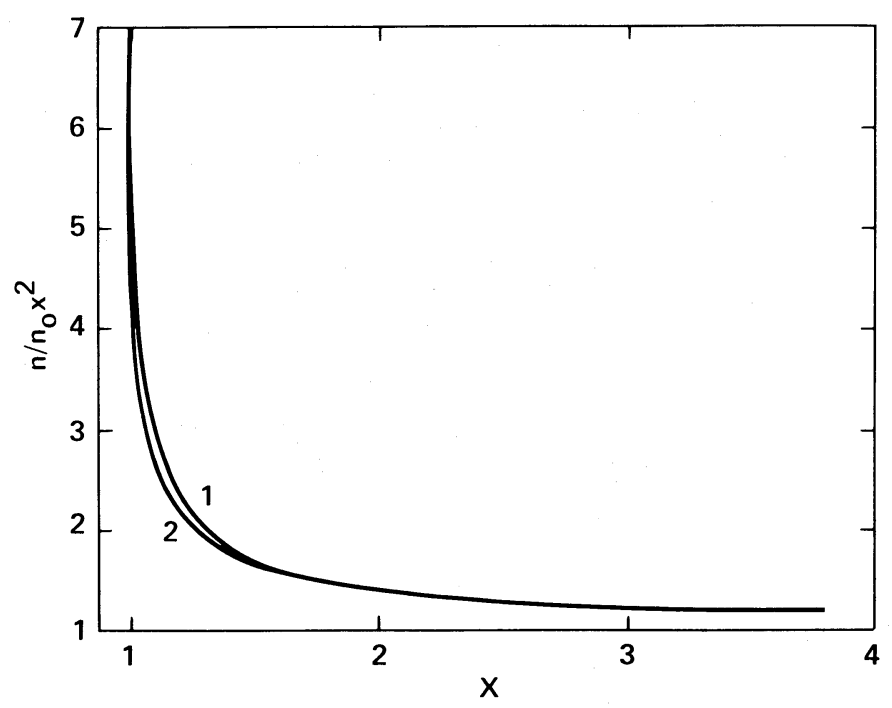

FIG. 14.-The analytical density distribution used in the model calculations of $\mathrm{OH} 26.5+0.6$ is compared to the second-order approximation using the local radiation field in the standard model to calculate the radiative acceleration. As in Fig. 3 the density has been divided by $n_{0}$ and multiplied by $x^{2}$ $\left(x=r / r_{0}\right)$ to emphasize the convergence to the results for a constant outflow velocity. For details see text.

shells, cf. $\S$ II $b$; Gillett et al. 1975) than in absorption toward Sgr A $(T \approx 10 \mathrm{~K} ;$ Roche and Aitken 1985).

Alternatively, this difference in the intrinsic silicate optical properties in the different sources might result from variations in the structure or composition of the condensing dust (Schutte and Tielens 1985; Nuth, Donn, and Nelson 1986). In particular, these differences may be the consequence of the increased temperature of the thermal equilibrium condensation zone of the silicates when the optical depth increases. In this picture, the radius of this zone (3-7 $r_{*}$ ) is set by the pulsations of the star rather than the thermal equilibrium condensation temperature of the silicate material. The temperature of the condensing silicates will then, in general, be much less than the thermal equilibrium dust condensation temperature, and a highly amorphous material will result (Tielens 1983; Tielens and Allamandola 1987b). A circumstellar dust shell with a larger optical depth will then have a higher temperature in the condensation zone than an optically thin envelope, due to back heating by the dust envelope. This will result in a more crystalline silicate structure. Laboratory experiments have shown that the higher the temperature of the grains, the more crystalline the material and the sharper the $10 \mu \mathrm{m}$ feature (Krätschmer and Huffman 1979). However, we note that our numerical models indicate very similar temperatures at the inner edge (i.e., $T_{s} \approx 1000 \mathrm{~K}$ ) for both $\mathrm{OH} 26.5+0.6$ and IRC 10011. This makes this second explanation perhaps less likely.

In Table 4 the mass-loss rates calculated from the model fits to the IR spectra of R Cas, IRC 10011, and OH/IR 26.5+0.6 are compared to the results from other determinations. These include (1) mass-loss determinations from millimeter $\mathrm{CO}$ lines, which assumes a $\mathrm{CO} / \mathrm{H}$ conversion factor; (2) submillimeter continuum measurements, which assume a dust emissivity per $\mathrm{H}$ atom; and (3) an estimate from the momentum equation. The latter is obtained by integrating the momentum equation of the gas, neglecting the gas pressure term and the gravitational force (see eq. [12]). This results in 


$$
\dot{M}=L_{*} \tau / c v_{\infty},
$$

where $\tau$ is the radiation pressure optical depth through the shell and $v_{\infty}$ the outflow velocity at large distance from the stellar surface (Salpeter 1974). Essentially, this equates the momentum in the wind with the momentum of absorbed photons. Neglecting scattering and frequency diffusion, $\tau$ is given by the near-infrared absorption optical depth, which is approximately the $10 \mu \mathrm{m}$ optical depth (cf. $\S \mathrm{Vc}$ ).

Apart from the dust-to-gas conversion factors, the most uncertain factor in the mass-loss rate determinations given in Table 4 is the distance. Model fits to observed IR spectra actually measure the optical depth through the circumstellar shell. Conversion to a mass-loss rate involves, however, the absolute value of the inner radius of the shell (see eq. [20]). Thus, although the observed IR flux depends on the distance squared, the derived mass-loss rate scales linearly with the distance. The other determinations are based on absolute intensity or luminosity measurements and depend therefore on the distance squared. The values quoted for the different massloss rates are all scaled to the distance of the source given in Table 3.

The hydrogen mass-loss rate derived from the IR observations of $\mathrm{R}$ Cas is about a factor of 3 less than that derived from the CO observations. This is too large to be solely attributable to the uncertainty in the distance for this well-known Mira variable. It is unlikely that we have overestimated the intrinsic strength of the $10 \mu \mathrm{m}$ silicate feature, $S(10 \mu \mathrm{m})$, since our estimate is in the low range for the strength of the silicate feature. Moreover, such a low $S(10 \mu \mathrm{m})$ would also imply that about $300 \%$ of the silicon is depleted in the form of silicates in the diffuse interstellar medium (see Tielens and Allamandola $1987 a$ ), which is uncomfortably high. A low silicon depletion fraction in R Cas is also unlikely, since analysis of the thermal $\mathrm{SiO}$ emission from this object (Morris et al. 1979) suggests that only $5 \%-13 \%$ of the available silicon is in the form of the dominant $\mathrm{Si}$-bearing species in the gas phase, $\mathrm{SiO}$ (see Table 4). Probably, we have underestimated the gas mass-loss rate, since, for such a low mass-loss rate the coupling between gas and dust is small, and only a small fraction of the available photon momentum is transferred to the gas. Indeed, the dustgas drift velocity is calculated to be about $10 \mathrm{~km} \mathrm{~s}^{-1}$ for these conditions (Tielens 1983). Although this is contrary to our assumptions in deriving the gas density distribution, this has little influence on the dust density distribution and thus the emergent IR spectrum (see Appendix Ac). However, a large dust-gas drift velocity will increase our estimated dust massloss rate and thus also the gas mass-loss rate. Note that for the same reason the mass-loss rate estimated using the momentum equation (eq. [22]) is much larger than that calculated from the IR spectrum or the $\mathrm{CO}$ data.

The hydrogen mass-loss rate calculated from the IR and from CO observations of IRC 10011 are in reasonable agreement, indicating that in this source all the available silicon has condensed out in the form of silicate grains (see Table 4). Indeed, thermal $\mathrm{SiO}$ studies indicate that $99 \%$ of the silicon has condensed out (Morris et al. 1979). The dust mass-loss rates estimated from the submillimeter dust continuum agree also very well with the IR one, suggesting that the assumed submillimeter emission efficiency is correct. We note that the ratio of submillimeter efficiency to the peak $10 \mu \mathrm{m}$ strength in our model calculations is somewhat larger (factor of $\sim 2$ ) than indicated by this comparison. This will, however, not affect our results. The mass-loss rate estimated from the momentum equation is in reasonable agreement with the IR result given the approximations involved in deriving it (e.g., neglecting gravitational attraction; $Q_{r p}=Q_{\text {peak }}$ ).

The IR mass-loss rate of $\mathrm{OH} 26.5+0.6$ is again in reasonable agreement with that estimated from the momentum equation. Note that many previously published estimates based on equation (22) essentially assumed that frequency diffusion of IR photons is rapid, implying that $\tau$ is of order unity rather than $\tau(10 \mu \mathrm{m}) \approx 30$. A quantum of energy is, however, absorbed and reemitted many, many times before it reaches such low frequencies that the envelope is optically thin and it escapes (see $\S \mathrm{Vc}$ ). Consequently, this is not a good approximation. Taken at face value, the $\mathrm{CO}$ results would suggest that the mass-loss rate of the heavily obscured object $\mathrm{OH} 26.5+0.6$ is comparable to that of the optically thin shell around R Cas. The IR results, however, show otherwise. In this respect we note that CO is hard to detect in many OH/IR stars (van der Veen 1987). Since the CO data reflect the condition at the very outer edge of the shell $\left(\sim 3 \times 10^{16} \mathrm{~cm}\right)$, while the mid-IR originates slightly deeper $\left(\sim 10^{16} \mathrm{~cm}\right)$, this might indicate a very recent increase in mass-loss rate. Such a rapid increase in mass-loss rate might be connected to the superwind which starts the protoplanetary nebula phase (Kwok, Purton, and Fitzgerald 1978). Clearly, until this discrepancy is resolved, one should be careful with any $\mathrm{CO}$ mass-loss rates. Finally, it is worth noting that the phase of intense mass-loss represented by $\mathrm{OH} 26.5+0.6$ has to

TABLE 4

MASS-LOSS RATES ${ }^{\mathbf{a}}$

\begin{tabular}{|c|c|c|c|c|c|c|c|c|}
\hline \multirow[b]{2}{*}{ Овјест } & \multicolumn{2}{|c|}{ THIS WORK } & \multicolumn{2}{|c|}{ CO DATA ${ }^{b}$} & \multicolumn{2}{|c|}{$\mathrm{SiO} \mathrm{DATA}^{\mathrm{c}}$} & \multirow{2}{*}{$\begin{array}{c}\operatorname{SuBMM}^{\mathrm{d}} \\
\dot{M}_{d}\end{array}$} & \multirow{2}{*}{$\begin{array}{c}\text { MOMENTUM } \\
\dot{M}_{\mathrm{H}}\end{array}$} \\
\hline & $\dot{M}_{d}$ & $\dot{M}_{\mathrm{H}}$ & $\dot{M}_{\text {co }}$ & $\dot{M}_{\mathrm{H}}$ & $\dot{M}_{\text {SiO }}$ & $\dot{M}_{\mathrm{H}}$ & & \\
\hline $\mathrm{R}$ Cas $\ldots . .$. & $1.2(-9)$ & $2.9(-7)$ & $3.6(-9)$ & $8.6(-7)$ & $5.6(-11)$ & $3.9(-8)$ & & $6.7(-6)$ \\
\hline IRC $10011 \ldots \ldots$. & $7.6(-8)$ & $1.9(-5)$ & $5.0(-8)$ & $1.2(-5)$ & $1.9(-10)$ & $1.3(-8)$ & $8.6(-8)$ & $4.6(-5)$ \\
\hline OH $26.5+0.6 \ldots$ & $8.8(-7)$ & $2.2(-4)$ & $1.5(-10)$ & $5.0(-7)$ & $\ldots$ & $\ldots$ & $\ldots$ & $5.4(-4)$ \\
\hline
\end{tabular}

a Mass-loss rates in units of $M_{\odot} \mathrm{yr}^{-1}$ scaled to the same distance (see Table 3).

b Taken from Knapp and Morris (1985) and van der Veen (1987), a [CO]/[H] abundance ratio of $3 \times 10^{-4}$ has been assumed.

c Taken from Morris et al. (1979), a [SiO]/[H] abundance ratio of $3.3 \times 10^{-5}$ has been assumed.

d Taken from Sopka et al. (1985) assuming a dust opacity at $400 \mu \mathrm{m}$ of $20 \mathrm{~cm}^{2} \mathrm{~g}^{-1}$. The dust absorption properties assumed in this paper yield $36 \mathrm{~cm}^{2} \mathrm{~g}^{-1}$ and thus the derived dust mass would be about half the value quoted here.

' Estimated assuming momentum conservation (i.e., $\dot{M}_{\mathrm{H}}=\tau L / v_{\infty} c$; Forrest et al. 1978; Werner et al. 1980), using the data in Table 3. The optical depth, $\tau$, has been set equal to the $10 \mu$ m optical depth. 
be short lived $\left(\lesssim 10^{4} \mathrm{yr}\right)$.

\section{SUMMARY}

We have studied the infrared emission of oxygen-rich latetype giants. The following conclusions can be drawn from this work.

1. The observed relation between the color temperature, $T_{c}$, and the strength of the $10 \mu \mathrm{m}$ band (in emission or absorption) reflects rather directly the ratio of the near-infrared to $10 \mu \mathrm{m}$ optical depth. This relation is therefore a good measure of the intrinsic near-infrared to $10 \mu \mathrm{m}$ absorption efficiency of circumstellar silicate grains.

2. The near-infrared absorption of circumstellar silicate grains is larger than expected on the basis of terrestrial minerals. This may be due to the presence of $\mathrm{Fe}^{2+}$ ions dissolved in the silicate matrix. Such color centers possess electronic transitions since the (partially filled) $d$ levels are split by the nonuniform electric field of neighboring ligands. The disordered structure of the silicates gives then rise to rather broad absorption features.

3. Using the derived ratio of the near-infrared to $10 \mu \mathrm{m}$ absorption efficiency, the $A_{10}-T_{c}$ relation can be calibrated in terms of the total dust column density and, thus, the mass-loss rate. Extensive radiative transfer calculations show that the derived mass-loss rates, in particular for the heavily obscured $\mathrm{OH} / \mathrm{IR}$ stars, are insensitive to variations in the other free parameters.

4. Detailed model fits have been made of the observed infrared spectra of three Miras: R Cas, IRC 10011, and $\mathrm{OH}$ $26.5+0.6$. The results show that the dust properties vary from source to source. In particular, the intrinsic shape of the $10 \mu \mathrm{m}$ absorption feature becomes progressively narrower when the dust shell becomes cooler. Such variations may be due to a temperature dependence of the optical constants or they may reflect differences in the condensation history of the circumstellar grains.

5. Mass-loss rates have been derived for these three sources and are compared to previous determinations. Some problems with mass-loss rates determined from a simple analysis of the momentum equation (e.g., $\dot{M}=\tau L_{*} / v_{\infty} c$ ) are pointed out.

A. G. G. M. Tielens acknowledges the hospitality of the Leiden Sterrewacht during a visit in 1983 September when this work was started. Willem Schutte thanks the Space Science Division at NASA Ames Research Center and the Space Sciences Laboratory at the University of California, Berkeley, for their generous hospitality during a short stay in 1986 August. We thank Mike Werner and David Hollenbach for critical reading of an earlier version of this manuscript and Wil van der Veen for communicating his $\mathrm{CO}$ mass-loss results for $\mathrm{OH}$ $26.5+0.6$ prior to publication.

\section{APPENDIX A}

\section{MODEL IR SPECTRA OF DUSTY CIRCUMSTELLAR SHELLS}

This appendix discusses the results of our free parameter study (see $\S$ III $d$ ).

\section{a) The Mass-Loss Rate}

Increasing the total dust column density increases the total flux in the 10 and $20 \mu \mathrm{m}$ emission features until the shell becomes optically thick at those wavelengths ( $\tau \gtrsim 3$; see Figure 15, the standard model). The 10 and $20 \mu \mathrm{m}$ emission features become more flat-topped, go over into self-absorption $(\tau \approx 3)$ and finally $(\tau \gtrsim 6)$ only an absorption feature remains (see Jones and Merrill 1976; Bedijn 1977). Because the dust condensation temperature is less than about $1000 \mathrm{~K}$, shortward of about $3 \mu \mathrm{m}$ the emergent flux is dominated by escaping stellar photons. For increasing dust column density the spectrum will appear cooler, due to the increased optical depth. The changes in the $2 \mu \mathrm{m}$ region are connected to those in the $10 \mu \mathrm{m}$ region by the ratio $Q_{0} / Q_{\text {peak }}$ (Jones and Merrill 1976; Bedijn 1977).

\section{b) The Absorption Properties of the Dust}

The ratio of the near-infrared absorption efficiency of the dust to that at $10 \mu \mathrm{m}, Q_{0} / Q_{\text {peak }}$, has a profound effect on the shape of the emergent spectra (Jones and Merrill 1976; Bedijn 1977). For optically thin models $(\tau[10 \mu \mathrm{m}] \lesssim 3)$, decreasing this ratio while keeping the total dust column density constant produces a smaller 10 and $20 \mu \mathrm{m}$ emission feature (see Figs. $15 a$ and $b$ ). Note that the inner boundary of the dust shell will move inward due to the boundary condition (see $\S$ II). As a result, although the temperature gradient is the same, the total mass of warm dust in the shell and therefore the emission in the 10 and $20 \mu \mathrm{m}$ features decreases, with decreasing $Q_{0} / Q_{\text {peak }}$.

For increasing dust column density (i.e., $\tau[10 \mu \mathrm{m}]>3$ ), the spectral differences around 10 and $20 \mu \mathrm{m}$ with the standard model with the same dust column density become less and less important (see Figs. 15a and $b$ ). Essentially, this is because the emission at these wavelengths is dominated by cool dust far out in the dust shell which is not directly heated by stellar photons (i.e., in the NIR) but by reemitted IR radiation (i.e., $\lambda \gtrsim 10 \mu \mathrm{m}$ ). Recall that the temperature of the warm dust will not change because of the inner boundary condition (i.e., $T\left[r_{0}\right]=T_{s}$; see $\S$ II). Of course, the near-infrared emission $(\lambda \lesssim 3 \mu \mathrm{m})$, which is dominated by escaping stellar photons, is strongly influenced by the ratio of the near-infrared to $10 \mu \mathrm{m}$ absorption cross section (see Figs. $15 a$ and $b$ ). For the same total dust column density (i.e., the same $10 \mu \mathrm{m}$ optical depth), a model with a smaller ratio will have smaller near-infrared absorption optical depth and thus a higher near-infrared color temperature. This effect is obviously more pronounced for the highly optically thick models. This effect can be used to determine the near-infrared absorption properties of circumstellar silicates from the observations (see § IV; Jones and Merrill 1976; Bedijn 1977). 

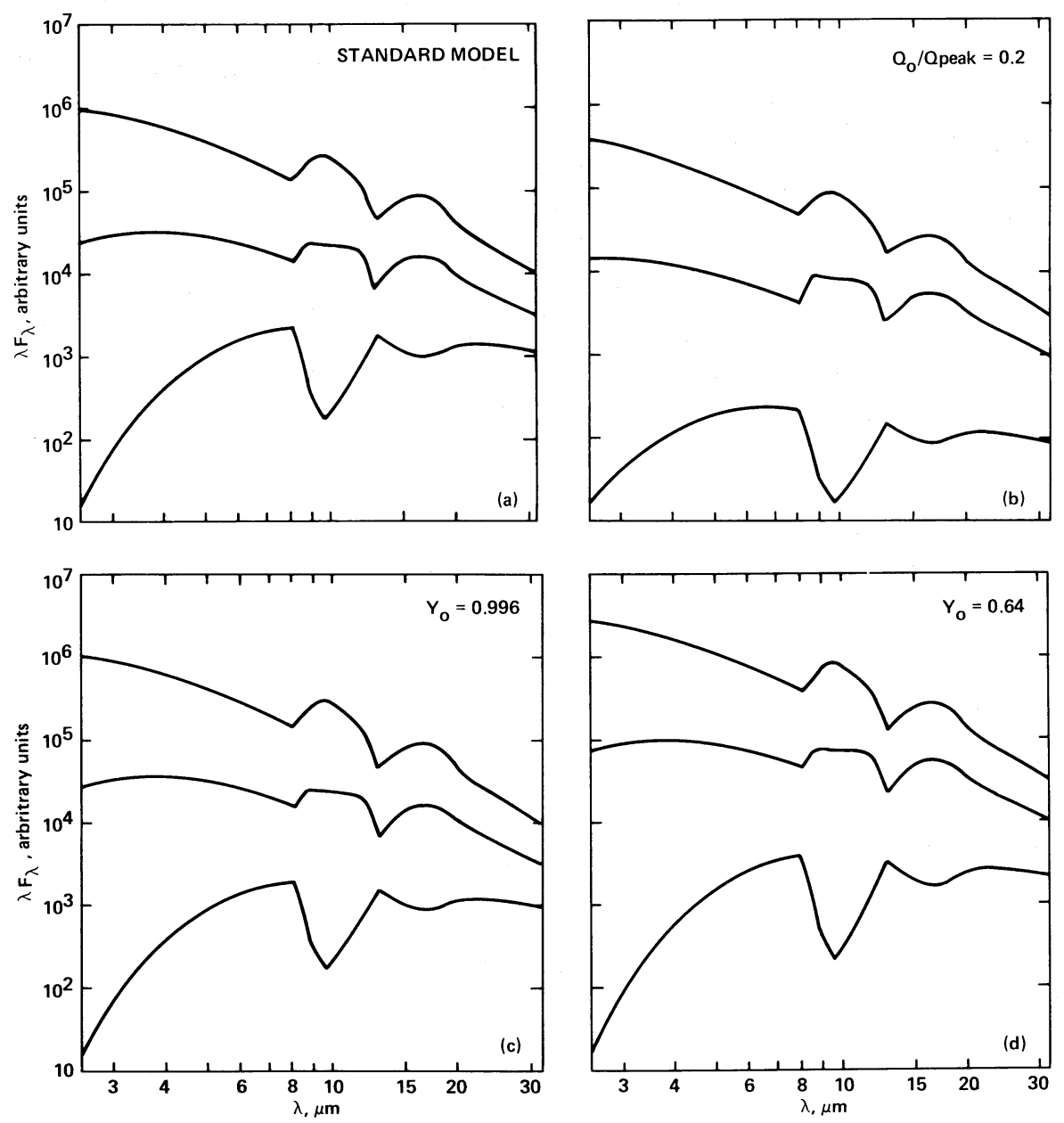

Fig. 15.-Emergent fluxes as a function of wavelength for three different $10 \mu \mathrm{m}$ optical depths, $0.3,3.0$, and 30 , respectively. The parameters for the standard model are given in Table 1. Each parameter has been varied independently: $(a)$ standard model; $(b)$ the ratio of near-infrared to $10 \mu \mathrm{m}$ opacity; $(c)$ and $(d)$ the density distribution in the acceleration zone; $(e)$ the dust condensation temperature; $(f)$ the stellar effective temperature; $(g)$ the ratio of the inner boundary to the stellar radius. The absolute flux levels at $2.2 \mu \mathrm{m}$ assuming $L_{*}=10^{4} L_{\odot}$ and $D=1 \mathrm{kpc}$ are given in Table 5 .

\section{c) The Dust Density Distribution}

The dust density distribution (i.e., $y_{0}$ ) has only minor influence on the emergent spectrum, in particular around $10 \mu \mathrm{m}$, if the total dust column density is kept constant (see Figs. $15 c$ and $d$ ). For larger $y_{0}$ the dust is more piled up at the inner boundary, and consequently the grains will be on the average hotter. However, since $10 \mu \mathrm{m}$ is on the Rayleigh- Jeans part of the blackbody curve for dust around $1000 \mathrm{~K}$, this produces only slight variations in the shape of the 10 (and $20 \mu \mathrm{m}$ ) feature. Of course, for optically thin models the envelope will emit more of the absorbed stellar energy at short wavelengths and less in the 10 and $20 \mu \mathrm{m}$ features.

TABLE 5

MODEL $2.2 \mu \mathrm{m}$ FLUX LEVELS

\begin{tabular}{ccccc}
\hline \hline & \multicolumn{4}{c}{$\lambda F_{\lambda}\left(2.2 \mu \mathrm{m}^{\mathrm{a}}\left(\mathrm{ergs} \mathrm{s}^{-1} \mathrm{~cm}^{-2}\right)\right.$} \\
\cline { 2 - 5 }$\tau(10 \mu \mathrm{m})$ & 0.3 & 3 & 30 & Figure $^{\mathrm{b}}$ \\
\hline Standard $\ldots \ldots \ldots$ & $1.09(-7)$ & $5.62(-8)$ & $1.65(-10)$ & $a$ \\
$Q_{0} / Q_{\text {peak }}=0.2 \ldots$. & $1.11(-7)$ & $8.27(-8)$ & $2.46(-9)$ & $b$ \\
$Y_{0}=0.996 \ldots \ldots \ldots$ & $1.09(-7)$ & $5.70(-8)$ & $2.00(-10)$ & $c$ \\
$Y_{0}=0.64 \ldots \ldots \ldots$ & $1.08(-7)$ & $5.44(-8)$ & $1.01(-10)$ & $d$ \\
$T_{\text {sub }}=750 \mathrm{~K} \ldots \ldots$. & $1.06(-7)$ & $4.85(-8)$ & $2.00(-11)$ & $e$ \\
$T_{*}=2700 \mathrm{~K} \ldots \ldots$. & $8.40(-8)$ & $4.90(-8)$ & $1.57(-10)$ & $f$ \\
$r_{\text {in }}=7 r_{*} \ldots \ldots \ldots$. & $1.04(-7)$ & $4.25(-8)$ & $1.17(-10)$ & $g$ \\
\hline
\end{tabular}

${ }^{a}$ Assuming luminosity $L_{*}=10^{4} L_{\odot}$ and distance $D=1 \mathrm{kpc}$.

b Shown in Fig. 15. 

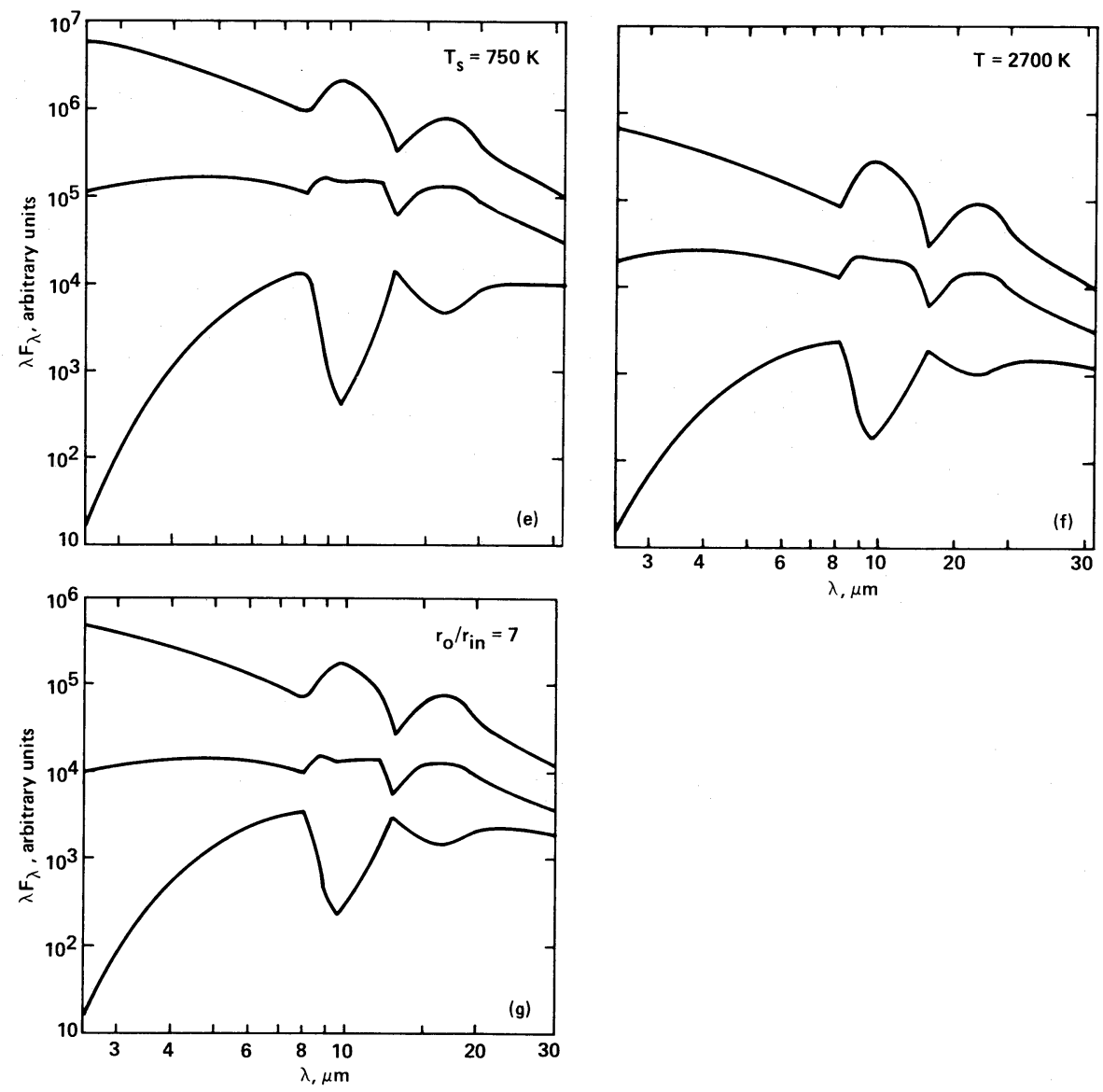

Fig. 15-Continued

However, varying $y_{0}$ from 0.9956 to 0.64 decreases the emission strength of the $10 \mu \mathrm{m}$ feature by at most $5 \%$. For large optical depths the spectra are identical, essentially because the emergent flux arises from regions far out in the shell, and the details of the dust density distribution around the inner radius are lost.

\section{d) The Dust Condensation Temperature}

A set of models, varying the total dust column density, have been run with a lower dust condensation temperature $\left(T_{s}=750 \mathrm{~K}\right.$; Fig. 15e). Note that since the absorption and emission properties of the dust are kept the same, this implies that the inner radius of the dust envelope moves outward. Generally, it can be said that, due to the overall lower dust temperature, the color temperature of the emitted radiation decreases. For an optically thin model the difference with the standard model with the same dust column density is generally small (see Figs. $15 a$ and $e$ ). Because of the lower average temperature there is somewhat more emission in the 10 and $20 \mu \mathrm{m}$ features and somewhat less in the near-infrared continuum $(\sim 3 \mu \mathrm{m})$. For the optically thick models the spectral differences become very pronounced. Because the average dust temperature in the circumstellar envelope is lower for the lower $T_{s}$ models, the 10 and $20 \mu \mathrm{m}$ emission features go over into self-absorption for smaller dust column densities. Likewise the 10 to $20 \mu \mathrm{m}$ features are stronger for the same column density (i.e., the $10 \mu \mathrm{m}$ optical depth). In the optically thick limit $(\tau[10 \mu \mathrm{m}] \gtrsim 30)$ spectra very similar to the standard model throughout the 10 and $20 \mu \mathrm{m}$ regions of the spectrum can be obtained by decreasing the total dust column density. Essentially, at these high optical depths, the emitted spectrum is dominated by the outer portions of the circumstellar shell. By decreasing the total dust column density, the temperature and density structure in the outer regions can be made to agree with that of the standard model. However, shortward of about $3.5 \mu \mathrm{m}$ the emergent flux is dominated by escaping stellar photons and by emission from the hot inner shell regions. These are strongly influenced by the total dust column density and the condensation temperature at the inner radius.

\section{e) The Stellar Parameters}

The value chosen for the total luminosity of the central star has no influence on the shape of the emergent spectrum when the total dust column density is kept constant (Jones and Merrill 1976; Bedijn 1977). This is implicit in our assumption that the inner radius of the circumstellar shell is set by the condition that the dust temperature is equal to the condensation temperature. In other words a certain flux has to pass through the inner boundary. Obviously, the absolute dimensions of the shell are determined by the total luminosity.

Keeping all other parameters constant, a hotter star will radiate relatively more at shorter wavelengths than a cooler star. Because 
of the increase of the absorption efficiency with frequency (see eq. [6]), this is similar to changing the ratio of the near-infrared to $10 \mu \mathrm{m}$ absorption efficiency. Recall that the inner boundary condition (i.e., $T_{d}\left[r_{0}\right]=T_{s}$ ) implies that the dust shell will move out. For the same dust column density, this results in a larger dust mass in the shell. In the optically thin limit $(\tau[10 \mu \mathrm{m}] \lesssim 3)$ the flux in the 10 and $20 \mu \mathrm{m}$ emission feature will therefore increase (see Figs. $15 a$ and $f$ ). The variations in the total flux in the 10 and $20 \mu \mathrm{m}$ features due to variations in the stellar effective temperature can be roughly compensated for by keeping the product $T_{\text {eff }} \tau(10 \mu \mathrm{m}) Q_{0} / Q_{\text {peak }}$ constant (i.e., the total flux absorbed by the shell). Shortward of about $3 \mu \mathrm{m}$ the emission is dominated by escaping stellar photons and changes thus considerably with the stellar effective temperature. Thus, even when the total flux in the 10 and $20 \mu \mathrm{m}$ features is kept constant, the contrast with the (stellar) continuum will vary.

In the optically thick limit $(\tau[10 \mu \mathrm{m}] \gtrsim 3)$, the spectra longward of $6 \mu \mathrm{m}$ become more or less independent of the effective temperature of the star (see Figs. $15 a$ and $f$ ). Essentially this is because the spectrum is now dominated by emission from cool dust grains which are shielded from the stellar photons, and their main heating source (warm dust grains closer to the star) does not vary due to the inner boundary condition. Even at the inner boundary backheating by IR photons emitted by the dust shell dominates over direct heating by stellar photons. Thus the density and temperature structure are almost independent of the stellar effective temperature. Shortward of about $3 \mu \mathrm{m}$ the stellar flux dominates the emergent spectrum, and this spectral region will thus depend on the effective temperature.

\section{f) The Inner Radius}

The ratio of the inner dust shell radius to the stellar radius, $r_{0} / r_{*}$, is fully fixed when the free parameters listed above have been chosen. However, the onset of dust condensation might be dominated by the decay of the shock wave activity in the extended stellar photosphere, rather than the condensation temperature of the dust (see $\S$ II $a$ ). Taking $r_{0} / r_{*}$, rather than $T_{s}$, fixed between models, the dust condensation temperature will increase continuously with increasing dust column density, as backheating by the circumstellar envelope becomes more and more important. For $r_{0} / r_{*}=7, T_{s}$ is 615,710 , and $930 \mathrm{~K}$ for $\tau=0.3,3$, and 30, respectively. The variations in the emergent spectrum are thus very similar to those introduced by varying the condensation temperature ( $\S d$; see Figs. 15a,e, and $g$ ).

\section{REFERENCES}

Allen, C. W. 1973, Astrophysical Quantities, (London: Athlone).

Allen, D. A., Barton, J. R., Gillingham, P. R., and Phillips, B. A. 1980 , M.N.R.A.S., 190, 531.

Bamford, C. R. 1962, Phys. Chem. Glasses, 3, 189.

Bates, T. 1962, in Modern Aspects of the Vitreous State, Vol. 2, ed. J. D. Mackenzie (London: Butterworths), p. 195.

Baud, B., Sargent, A. I., Werner, M. W., and Bentley, A. F. 1985, Ap. J., 292, 628.

Becklin, E. E., Mathews, K., Neugebauer, G., and Willner, S. P. 1978, Ap. J., 220, 831 .

Beckwith, S. 1985, in Mass Loss from Red Giants, ed. M. Morris and B. Zuckerman (Dordrecht: Reidel), p. 95 .

Bedijn, P. 1977, Ph.D. thesis, Leiden.

. 1987, Astr. Ap., 186, 136.

Bishay, A., and Kinawi, A. 1965, in Physics of Non-crystalline Solids, ed. J. A Prim (New York: Wiley), p. 589.

Bowen, G. H., and Beach, T. E. 1987, in Late Stages of Stellar Evolution, ed. S. Kwok, and S. R. Pottasch (Dordrecht: Reidel), p. 275.

Bowen, G. H. 1988, Ap. J., 329, 299.

Campbell, M. F., et al. 1976, Ap. J., 208, 396.

Cohen, J. G., and Frogel, J. A. 1977, Ap. J., 211, 178

Cohen, M. 1981, Pub. A.S.P., 93, 288.

Day, K. L. 1976, Ap. J., 210,614.

. 1979, Ap. J., 234, 158.

Day, K. L., and Donn, B. 1978, Ap. J. (Letters), 222, L45.

Draine, B. T., and Lee, H. M. 1984, Ap. J. 285, 89.

Dwek, E., and Scalo, J. M. 1980, Ap. J., 239, 193.

Dyck, H. M., Lockwood, G. W., and Capps, R. W. 1974, Ap. J., 189, 89.

Dyck, H. M., Zuckerman, B., Leinert, Ch., and Beckwith, S. 1984, Ap. J., 287, 801.

Edwards, R. J., Paul, A., and Douglas, R. W. 1972, Phys. Chem. Glasses, 13, 131.

Engels, D. 1982, Veröff. Astr. Inst. Bonn, Nr. 95.

Evans, N., and Beckwith, S. 1977, Ap. J., 217, 729.

Fanderlik, I. 1983, Glass Science and Technology, Vol. 5(Amsterdam: Elsevier).

Farrell, E. F., and Newnham, R. E. 1965, American Mineralogist, 50, 1972.

Feast, M. W., Catchpole, R. M., Whitelock, P. A., Roberts, G., Spencer Jones, J., and Carter, B. S. 1983, M.N.R.A.S., 203, 1207.

Fix, J. D., and Cobb, M. L. 1988, Ap. J., 329, 290.

Forrest, W. J., Gillett, F. C., and Stein, W. A. 1975, Ap. J., 195, 423.

Forrest, W. J., et al. 1978, Ap. J., 219, 114.

Forrest, W. J., McCarthy, J. F., and Houck, J. R. 1979, Ap. J., 233, 611.

Gal, O., de Muizon, M., Papoular, R., and Pégourie, B. 1987, Astr. Ap., 183, 29.

Gatley, I., Becklin, E. E., Werner, M. W., and Wynn Williams, C. G. 1977, Ap. J., 216, 277.

Gehrz, R. D., Kleinmann, S. G., Mason, S., Hackwell, J. A., and Grasdalen, G. L. 1985, Ap. J., 290, 296.
Gillett, F. C., Forrest, W. J., Merrill, K. M., Capps, R. W., and Soifer, B. T. 1975, Ap. J., 200, 609.

Gilman, R. C. 1972, Ap. J., 178, 423.

Greenberg. J. M. 1968, in Stars and Stellar Systems Vol 7: Nebulae and Interstellar Matter, ed. B. M. Middlehurst and L. W. Aller (University of Chicago Press), p.221.

Hackwell, J. A. 1971, Ph. D. thesis, University College, London.

Hayes, D. S., Mavko, G. E., Radick, R. R., Rex, K. H., and Greenberg, J. M. 1973, in Interstellar Dust and Related Topics, ed. J. M. Greenberg and H. C. van de Hulst, (Dordrecht: Reidel), p. 83

Herman, J. 1983, Ph.D. thesis, University of Leiden.

Hinkle, K. H. 1985, in Mass Loss from Giants, ed. M. Morris and B. Zuckerman (Dordrecht: Reidel), p. 85

Hinkle, K. H., Hall, D. N. B., and Ridgway, St. T. 1982, Ap. J., 252, 697

Hinkle, K. H., Scharlach, W. W. G., and Hall, D. N. B. 1984, Ap. J. Suppl., 56, 1.

Hyland, A. R., Becklin, E. E., Frogel, J. A., and Neugebauer, G. 1972, Astr. Ap., $16,204$.

IRAS Science Team. 1986, Astr. Ap. Suppl., 65, 607.

Jewell, P. R., Webber, J. C., and Snyder, L. E. 1980, Ap. J. (Letters), 242, L29.

Jones, T. W., and Merrill, K. M. 1976, Ap. J., 209, 509.

Jura, M. 1984, Ap. J., 282, 200.

1987, in Interstellar Processes, ed. D. Hollenbach and H. Thronson (Dordrecht: Reidel), p. 3

Knapp, G. R., and Morris, M. 1985, Ap. J., 292, 640

Kozasa, T., and Hasegawa, H. 1988, Icarus, 73, 180.

Krätschmer, W., and Huffman, D. R. 1979, Astr. Space Sci., 61, 195.

Kwan, J., and Scoville, N. 1976, Ap. J., 209, 102.

Kwok, S. 1975, Ap. J., 198, 583.

Kwok, S., Purton, C. R., and FitzGerald, P. M. 1978, Ap. J. (Letters), 219, L125.

Le Bertre, T. 1987, Astr. Ap., 180, 160.

Leung, C. M. 1975, Ap. J., 199, 340.

Leung, . M76a, J. Quant. Spectros. Rad. Tranf., 16, 559. 1976b, Ap. J., 209, 75.

Martin, P. G., and Rogers, C. 1987, Ap. J., 322, 374.

Mathis, J. S., Rumpl, W., and Nordsieck, K. H. 1977, Ap. J., 217, 425

McCarthy, J. F., Forrest, W. J., Briotta, D. A., and Houck, J. R. 1980, Ap. J., 242, 965 .

McKee, C. F., Hollenbach, D., Seab, C. G., and Tielens, A. G. G. M. 1987, Ap. J., 318, 674 .

Merrill, K. M. 1977, in The Interaction of Variable Stars with Their Environment, ed. R. Kippenhahn, J. Rahe, and W. Stronmeier (Veröff. Remeis Stern. Bamberg, Vol. 11, No. 121), p. 446.

Merrill, K. M., and Stein, W. A. 1976a, Pub. A.S.P., 88, 285.

.1976b, Pub. A.S.P., 88, 294.

Mitchell, R. M., and Robinson, G. 1981, M.N.R.A.S., 196, 801

Morris, M., Redman, R., Reid, M. J., and Dickinson, D. F. 1979, Ap. J., 229, 75. 
Nguyen-Q-Rieu, Laury-Micoulaut, C., Winnberg, A., and Schultz, G. V. 1979, Astr. Ap., 75, 351 .

Nuth, J. A., and Donn, B. 1985, Ap. J., 288, 187.

Nuth, J. A., Donn, B., and Nelson, R. 1986, Ap. J. (Letters), 310, L83.

Olnon, F. M. 1979, Ph.D. thesis, University of Leiden.

Pégourie, B., and Papoular, R. 1985, Astr. Ap., 142, 451

Penman, J. M. 1976, M.N.R.A.S., 175, 149.

Plendl, J. N. 1970, in Far-Infrared Properties of Solids, ed. S. S. Mitra and S. Nudelman (New York: Plenum), p. 387.

Roche, P. F., and Aitken, D. K. 1985, M.N.R.A.S., 215, 425.

Rogers, C., Martin, P. G., and Crabtree, D. R. 1983, Ap. J., 272, 175

Rogers, C., and Martin, P. G. 1984, Ap. J., 284, 327.

Rowan-Robinson, M., and Harris, S. 1982, M.N.R.A.S., 200, 197. 1983, M.N.R.A.S., 202, 767.

Salpeter, E. E. 1974, Ap. J., 193, 585.

Schulman, J. H., and Compton, W. D. 1962, Color Centers in Solids, (New York: Pergamon)

Schutte, W., and Tielens, A. G. G. M. 1985, in Mass Loss from Red Giants, ed. M. Morris and B. Zuckerman (Dordrecht:Reidel), p. 89.

Seab, G. G. 1987, in Interstellar Processes, ed. D. Hollenbach and H. Thronson (Dordrecht: Reidel), p. 491.

Simpson, J., and Rubin, R. 1989, in Interstellar Dust, Contributed Papers, ed. L. J. Allamandola and A. G. G. M. Tielens (NASA-CP) in press.

Sopka, R. J., Hildebrand, R., Jaffe, D. T., Gatley, I., Roellig, T., Werner, M., Jura, M., and Zuckerman, B. 1985, Ap. J., 294, 242.

Steele, S. T., and Douglas, R. W. 1965, Phys. Chem. Glasses, 6, 112

Strecker, D. W., Erickson, E. F., and Witteborn, F. C. 1978, A.J., 83, 26.

Taam, R. E., and Schwartz, R. D. 1976, Ap. J., 204, 842.

Tielens, A. G. G. M. 1983, Ap. J., 271, 702. .1989, in preparation.
Tielens, A. G. G. M., and Allamandola, L. J. 1987a, in Interstellar Processes, ed. D. Hollenbach and H. Thronson (Dordrecht: Reidel), p. 397.

. 1987b, in Physical Processes in Interstellar Clouds, ed. G. Morfill and M. Scholer (Dordrecht: Reidel), p. 333.

Tielens, A. G. G. M., Werner, M. W., and Capps, R. 1985, in Mass Loss from Red Giants, ed. M. Morris, and B. Zuckerman (Dordrecht: Reidel), p. 305

Thronson, H. A., and Harper, D. A. 1979, Ap. J., $230,133$.

van de Hulst, H. C. 1957, Light Scattering by Small Particles, (NY: Wiley).

van der Veen, W., Habing, H. J., and Geballe, T. R. 1987, in Planetary and Protoplanetary Nebulae: from IRAS to ISO, ed. A. Preite-Martinez (Dordrecht: Reidel), p. 69.

van der Veen, W. E. C. J. 1987, private communication.

Volk, K., and Kwok, S. 1988, Ap. J., 331, 435.

Wallerstein, G. 1977, R.A.S.C. Journal, 71, 298.

Werner, M. W., Beckwith, S., Gatley, I., Sellgren, K., Berriman, G., and Whiting, D. L. 1980, Ap. J., 239, 540.

Willems, F. J., and de Jong, T. 1982, Astr. Ap., 115, 213.

Willson, L. A. 1976, Ap. J., 205, 172 .

. 1987, in Late Stages of Stellar Evolution, ed. S. Kwok and S. R. Pottasch, (Dordrecht: Reidel), p. 253

Willson, L. A., and Hill, S. J. 1979, Ap. J., 228, 854.

Wong, J., and Angell, C. A. 1976, Glass Structure by Spectroscopy (NY: Dekker).

Wood, P. R. 1979, Ap. J., 227, 220.

Wooten, B. J. 1972, Optical Properties of Solids, (New York: Academic). Zappala, R. R., Becklin, E. E., Matthews, K., and Neugebauer, G. 1974, Ap. J.,
192, 109.

W. SchutTE: Space Sciences Division, NASA Ames Research Center, MS: 245-6, Moffett Field, CA 94035

A. G. G. M. TIELENS: Space Sciences Division, MS: 245-3, NASA Ames Research Center, Moffett Field, CA 94035 